\title{
General Synthesis of (Salen)ruthenium(III) Complexes via N...N Coupling of (Salen)ruthenium(VI) Nitrides
}

\author{
Wai-Lun Man, ${ }^{\dagger}$ Hoi-Ki Kwong, ${ }^{\dagger}$ William W. Y. Lam, ${ }^{\dagger}$ Jing Xiang, ${ }^{\dagger}$ Tsz-Wing Wong, ${ }^{\dagger}$ Wing-Hong Lam, ${ }^{\dagger}$ \\ Wing-Tak Wong, ${ }^{\ddagger}$ Shie-Ming Peng, ${ }^{\S}$ and Tai-Chu Lau ${ }^{*, t}$ \\ Contribution from the Department of Biology and Chemistry, City University of Hong Kong, Tat \\ Chee Avenue, Kowloon Tong, Hong Kong, China, Department of Chemistry, University of Hong \\ Kong, Pokfulam Road, Hong Kong, China, and Department of Chemistry, National Taiwan \\ University, Taipei 106, Taiwan
}

Received February 13, 2008

\begin{abstract}
Reaction of $\left[\mathrm{Ru}^{\mathrm{Vl}}(\mathrm{N})\left(\mathrm{L}^{1}\right)(\mathrm{MeOH})\right]^{+}\left(\mathrm{L}^{1}=N, N^{\prime}\right.$-bis(salicylidene)-o-cyclohexylenediamine dianion) with excess pyridine in $\mathrm{CH}_{3} \mathrm{CN}$ produces $\left[\mathrm{Ru}^{\prime \prime \prime}\left(\mathrm{L}^{1}\right)(\mathrm{py})_{2}\right]^{+}$and $\mathrm{N}_{2}$. The proposed mechanism involves initial equilibrium formation of $\left[\mathrm{Ru}^{\mathrm{VI}}(\mathrm{N})\left(\mathrm{L}^{1}\right)(\text { py })\right]^{+}$, which undergoes rapid $\mathrm{N} \cdots \mathrm{N}$ coupling to produce $\left[(p y)\left(\mathrm{L}^{1}\right) \mathrm{R} u^{\prime \prime \prime} N \equiv N-R u^{\prime \prime \prime}\left(\mathrm{L}^{1}\right)(\mathrm{py})\right]^{2+}$; this is followed by pyridine substituion to give the final product. This ligand-induced $\mathrm{N} \cdots \mathrm{N}$ coupling of $\mathrm{Ru}=\mathrm{N}$ is utilized in the preparation of a series of new ruthenium(III) salen complexes, $\left[R u^{\prime \prime \prime}(L)(X)_{2}\right]^{ \pm}\left(L=\right.$ salen ligand; $X=H_{2} O$, 1-Melm, py, $\mathrm{Me}_{2} \mathrm{SO}, \mathrm{PhNH}_{2},{ }^{\mathrm{B}} \mathrm{BuNH}_{2}, \mathrm{Cl}^{-}$or $\left.\mathrm{CN}^{-}\right)$. The structures of $\left[\mathrm{Ru}^{\prime \prime \prime}\left(\mathrm{L}^{1}\right)\left(\mathrm{NH}_{2} \mathrm{Ph}\right)_{2}\right](\mathrm{PF} 6)(6), \mathrm{K}_{6}\left[\mathrm{Ru}^{\prime \prime \prime}\left(\mathrm{L}^{1}\right)(\mathrm{CN})_{2}\right]$ (9), $\left[\mathrm{Ru}^{\prime \prime \prime}\left(\mathrm{L}^{2}\right)\left(\mathrm{NCCH}_{3}\right)_{2}\right]\left[\mathrm{Au}^{\prime}(\mathrm{CN})_{2}\right](11)\left(\mathrm{L}^{2}=N, N^{\prime}\right.$-bis(salicylidene)-o-phenylenediamine dianion) and [ $\left.\mathrm{N}^{n} \mathrm{Bu}_{4}\right]\left[\mathrm{Ru}^{\prime \prime \prime}\left(\mathrm{L}^{3}\right) \mathrm{Cl}_{2}\right]$ (12) $\left(\mathrm{L}^{3}=N, N^{\prime}\right.$-bis(salicylidene)ethylenediamine dianion) have been determined by $\mathrm{X}$-ray crystallography.
\end{abstract}

\section{Introduction}

Metal complexes bearing salen-type ligands have played important roles in the development of coordination chemistry for over half a century. In particular, manganese salen complexes have been widely used as catalysts for alkene epoxidation. $^{1-7}$ Recently, a number of ruthenium salen complexes have also been found to be active catalysts in

* To whom correspondence should be addressed: Email: bhtclau@ cityu.edu.hk.

City University of Hong Kong.

$\doteqdot$ University of Hong Kong.

$\S$ National Taiwan University.

(1) Jacobsen, E. N. In Catalytic Asymmetric Synthesis; Ojima, I., Ed.; VCH: New York, 1993; pp 159-202.

(2) Jacobson, E. N. In Comprehensive Organometallic Chemistry II; Hegedus, L. S., Ed.; Elsevier: New York, 1995, pp 1097-1135.

(3) Jacobson, E. N.; Wu, M. H. In Comprehensive Asymmetric Catalysis II; Jacobson, E. N., Pfaltz, A., Yamamoto, H., Ed.; Springer: Berlin, 1999; pp 649-67.

(4) Katsuki, T. Coord. Chem. Rev. 1995, 140, 189-214.

(5) Katsuki, T. J. Mol. Catal. A. 1996, 113, 87-107.

(6) Samsel, E. G.; Srinivasan, K.; Kochi, J. K. J. Am. Chem. Soc. 1985, 107, 7606-7617.

(7) Srinivasan, K.; Michaud, P.; Kochi, J. K. J. Am. Chem. Soc. 1986, 108, 2309-2320.

(8) Li, G. Y.; Zhang, J.; Chan, P. W. H.; Xu, Z. J.; Zhu, N.; Che, C. M. Organometallics 2006, 25, 1676-1688.

(9) Miller, J. A.; Gross, B. A.; Zhuravel, M. A.; Jin, W.; Nguyen, S. T. Angew. Chem., Int. Ed. 2005, 44, 3885-3889. various organic transformations such as cyclopropanation, ${ }^{8-11}$ epoxidation, ${ }^{12-15}$ aziridination, ${ }^{16-18}$ sulfimidation, ${ }^{19-21}$ and Diels-Alder reactions ${ }^{22,23}$ (Scheme 1).

These catalysts have the general formula $[\mathrm{Ru}($ salen$)(\mathrm{X})-$ $(\mathrm{Y})]^{n+}$, where $\mathrm{X}$ or $\mathrm{Y}$ is a nitrosyl, ${ }^{12,23-33}$ carbonyl, ${ }^{16-21,34,35}$ phosphine, ${ }^{8,9,15,22,36-40}$ or pyridine group. ${ }^{10,11}$ However, there

(10) Miller, J. A.; Hennessy, E. J.; Marshall, W. J.; Scialdone, M. A.; Nguyen, S. T. J. Org. Chem. 2003, 68, 7884-7886.

(11) Miller, J. A.; Jin, W.; Nguyen, S. T. Angew. Chem., Int. Ed. 2002, 41, 2953-2956.

(12) Nakata, K.; Takeda, T.; Mihara, J.; Hamada, T.; Irie, R.; Katsuki, T. Chem.-Eur. J. 2001, 7, 3776-3782.

(13) Kureshy, R. I.; Khan, N. H.; Abdi, S. H. R. J. Mol. Catal. A. 1995, 96, 117-122.

(14) Kureshy, R. I.; Khan, N. H.; Abdi, S. H. R.; Bhatt, A. K. J. Mol. Catal. A. 1996, 110, 33-40.

(15) Leung, W. H.; Che, C. M. Inorg. Chem. 1989, 28, 4619-4622.

(16) Kawabata, H.; Omura, K.; Uchida, T.; Katsuki, T. Chem. Asian. J. 2007, 2, 248-256.

(17) Kawabata, H.; Omura, K.; Katsuki, T. Tetrahedron Lett. 2006, 47, 1571-1574.

(18) Omura, K.; Uchida, T.; Irie, R.; Katsuki, T. Chem. Commun. 2004, 2060-2061.

(19) Murakami, M.; Uchida, T.; Saito, B.; Katsuki, T. Chirality 2003, 15, $116-123$.

(20) Uchida, T.; Tamura, Y.; Ohba, M.; Katsuki, T. Tetrahedron Lett. 2003, 44, 7965-7968.

(21) Tamura, Y.; Uchida, T.; Katsuki, T. Tetrahedron Lett. 2003, 44, 33013303.

(22) Chow, C. P.; Shea, K. J. J. Am. Chem. Soc. 2005, 127, 3678-3679.

(23) Odenkirk, W.; Rheingold, A. L.; Bosnich, B. J. Am. Chem. Soc. 1992, 114, 6392-6398. 
Scheme 1. Examples of Ruthenium Salen Complexes as Active Catalysts in Organic Synthesis

$$
\begin{aligned}
& \text { 1. Cycloproponation } \\
& \text { 3. Aziridination } \\
& \text { 4. Sulfimidation } \\
& \text { 5. Diels-Alder reaction }
\end{aligned}
$$

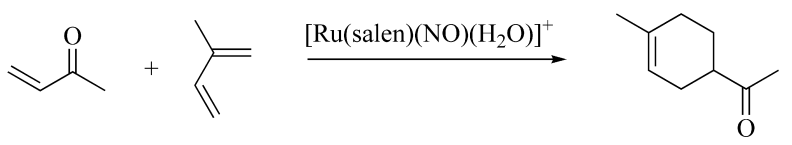

are no general methods for the preparation of these catalysts; each complex has to be prepared by a different method, as shown in eqs $1-4$.

$$
\begin{aligned}
& {\left[\mathrm{Ru}(\mathrm{NO}) \mathrm{Cl}_{3}\right] \underset{\mathrm{DMF}, 110{ }^{\circ} \mathrm{C}}{\stackrel{\text { salen}^{2-}}{\longrightarrow}}[\mathrm{Ru}(\text { salen })(\mathrm{NO}) \mathrm{Cl}]} \\
& {\left[\mathrm{Ru}\left(\mathrm{PPh}_{3}\right)_{3} \mathrm{Cl}_{2}\right] \underset{\text { refluxing MeOH }}{\stackrel{\text { salen2- }}{\longrightarrow}}\left[\mathrm{Ru}(\text { salen })\left(\mathrm{PPh}_{3}\right) \mathrm{Cl}\right]} \\
& {\left[\mathrm{Ru}(\text { salen })\left(\mathrm{PPh}_{3}\right)_{2}\right] \underset{\mathrm{CH}_{2} \mathrm{Cl}_{2}}{\stackrel{\mathrm{CO}}{\longrightarrow}}[\mathrm{Ru}(\text { salen })(\mathrm{CO})]} \\
& {\left[\mathrm{Ru}(\mathrm{p}-\text { cymene }) \mathrm{Cl}_{2}\right]_{2} \underset{\mathrm{LDA}, \mathrm{py}, \mathrm{THF}}{\stackrel{3,5-\mathrm{Bu}_{4} \text { salchda }^{2-}}{\longrightarrow}}[\mathrm{Ru}(3,5-} \\
& \left.{ }^{t} \mathrm{Bu}_{4} \text { salchda)(py) }{ }_{2}\right]
\end{aligned}
$$

We have reported that the ruthenium(VI) nitrido species, $\left[\mathrm{Ru}^{\mathrm{VI}}(\mathrm{N})\left(\mathrm{L}^{1}\right)(\mathrm{MeOH})\right]^{+}(\mathbf{1})$, can be readily prepared from $\left[\mathrm{N}^{n} \mathrm{Bu}_{4}\right]\left[\mathrm{Ru}^{\mathrm{VI}}(\mathrm{N}) \mathrm{Cl}_{4}\right]$. This species undergoes facile ligandinduced $\mathrm{N} \cdots \mathrm{N}$ coupling as represented by eq 5 ( $\mathrm{X}$ is a neutral ligand in this case). ${ }^{41}$

(24) Nakamura, Y.; Egami, H.; Matsumoto, K.; Uchida, T.; Katsuki, T. Tetrahedron 2007, 63, 6383-6387.

(25) Sun, W.; Yu, B.; Kühn, F. E. Tetrahedron Lett. 2006, 47, 1993-1996.

(26) Shimizu, H.; Onitsuka, S.; Egami, H.; Katsuki, T. J. Am. Chem. Soc. 2005, 127, 5396-5413.

(27) Egami, H.; Onitsuka, S.; Katsuki, T. Tetrahedron Lett. 2005, 46, 60496052.

(28) Irie, R.; Katsuki, T. Chem. Rec. 2004, 4, 96-109.

(29) Sauve, A. A.; Groves, J. T. J. Am. Chem. Soc. 2002, 124, 4770-4778.

(30) Bordini, J.; Hughes, D. L.; Da Motta Neto, J. D.; da Cunha, C. J. Inorg. Chem. 2002, 41, 5410-5416.

(31) Works, C. F.; Jocher, C. J.; Bart, G. D.; Bu, X.; Ford, P. C. Inorg. Chem. 2002, 41, 3728-3739.

(32) Works, C. F.; Ford, P. C. J. Am. Chem. Soc. 2000, 122, 7592-7593.

(33) Ellis, W. W.; Odenkirk, W. Chem. Commun. 1998, 1311-1312.

(34) Viswanathamurthi, P.; Natarajan, K. Transition Met. Chem. 1999, 24, 638-641.

(35) Khan, M. M. T.; Srinivas, D.; Kureshy, R. I.; Khan, N. H. Inorg. Chem. 1990, 29, 2320-2326.

(36) Syukri, S.; Sun, W.; Kühn, F. E. Tetrahedron Lett. 2007, 48, $1613-$ 1617.

(37) Venkatachalam, G.; Ramesh, R. Inorg. Chem. Commun. 2005, 8, 10091013.

(38) Ramesh, R. Inorg. Chem. Commun. 2004, 7, 274-276.

(39) Liang, J. L.; Yu, X. Q.; Che, C. M. Chem. Commun. 2002, 124-125.

$$
\begin{array}{r}
{\left[\mathrm{Ru}^{\mathrm{VI}}(\mathrm{N})\left(\mathrm{L}^{1}\right)(\mathrm{MeOH})\right]^{+}+2 \mathrm{X} \rightarrow\left[\mathrm{Ru}^{\mathrm{IIII}}\left(\mathrm{L}^{1}\right)(\mathrm{X})_{2}\right]^{+}+} \\
\mathrm{MeOH}+\frac{1}{2} \mathrm{~N}_{2}
\end{array}
$$

In this work, we make use of this $\mathrm{N} \cdots \mathrm{N}$ coupling reaction to synthesize a series of new ruthenium(III) complexes bearing various salen ligands that are potential catalysts for organic transformations. The structures of the salen ligands used in this work are shown in Figure 1.

\section{Experimental Section}

Materials. $\left[\mathrm{Ru}^{\mathrm{VI}}(\mathrm{N})\left(\mathrm{L}^{1}\right)(\mathrm{MeOH})\right]\left(\mathrm{PF}_{6}\right)(\mathbf{1}),{ }^{41}\left[\mathrm{~N}^{n} \mathrm{Bu}_{4}\right]\left[\mathrm{Ru}^{\mathrm{VI}}(\mathrm{N})-\right.$ $\left.\mathrm{Cl}_{4}\right]{ }^{42}$ and $\left[\mathrm{Ru}^{\mathrm{III}}\left(\mathrm{L}^{1}\right)\left(\mathrm{OH}_{2}\right)_{2}\right]\left(\mathrm{PF}_{6}\right)^{43}(2)$ were prepared by literature procedures. The Schiff base ligands, $\mathrm{H}_{2} \mathrm{~L}^{1}, \mathrm{H}_{2} \mathrm{~L}^{2}$, and $\mathrm{H}_{2} \mathrm{~L}^{3}\left(\mathrm{~L}^{1}, \mathrm{~L}^{2}\right.$ and $\mathrm{L}^{3}=N, N^{\prime}$-bis(salicylidene)-o-cyclohexylenediamine, $N, N^{\prime}$ bis(salicylidene)-o-phenylenediamine, and $N, N^{\prime}$-bis(salicylidene)ethylenediamine dianion respectively) were synthesized by condensation of salicylaldehyde with the corresponding diamines in refluxing ethanol. ${ }^{n} \mathrm{Bu}_{4} \mathrm{NPF}_{6}$ (Aldrich) was recrystallized three times from boiling ethanol and dried in vacuo at $120{ }^{\circ} \mathrm{C}$ for one day. Acetonitrile (Aldrich) for electrochemistry was distilled over calcium hydride. All other chemicals were of reagent grade and used without further purification. All manipulations were performed without precaution to exclude air or moisture unless otherwise stated.

Physical Measurements. IR spectra were obtained as $\mathrm{KBr}$ discs using a Nicolet 360 FTIR spectrophotometer. ${ }^{1} \mathrm{H}$ NMR spectra were recorded on a Varian $(300 \mathrm{MHz})$ FT NMR spectrometer. The chemical shifts (ppm) were reported with reference to tetramethylsilane (TMS). UV-vis spectra were recorded with a PerkinElmer Lamda 19 spectrophotometer in $1 \mathrm{~cm}$ quartz cuvettes. Elemental analysis was performed using an Elementar Vario EL Analyzer. Magnetic measurements were performed at room temperature using a Sherwood magnetic balance (Mark II). Conductivity measurements were done with a Cole-Parmer 01481-61 conductivity meter. Electrospray ionization mass spectrometry (ESI-MS) were performed with a PE-SCIEX API 300 triple quadruple mass spectrometer. Cyclic voltammetry measurements were performed with a PAR model 273 potentiostat using a glassy carbon working electrode, a $\mathrm{Ag} / \mathrm{AgNO}_{3}\left(0.1 \mathrm{M}\right.$ in $\left.\mathrm{CH}_{3} \mathrm{CN}\right)$ reference electrode, a platinum wire counter electrode with ferrocene $\left(\mathrm{FeCp}_{2}\right)$ as the internal standard.

Preparations. $\left[\mathbf{R u}^{\mathrm{III}}\left(\mathrm{L}^{1}\right)(\mathbf{1}-\mathrm{MeIm})_{2}\right]\left(\mathbf{P F}_{6}\right)(3)$. A green solution of $\mathbf{1}(120 \mathrm{mg}, 0.20 \mathrm{mmol})$ in 1-methylimidazole $(2 \mathrm{~mL})$ was stirred for 1 day. Slow addition of diethyl ether $(50 \mathrm{~mL})$ produced a green precipitate, which was filtered and then air-dried. Yield: (82\%). IR $\left(\mathrm{KBr}, \mathrm{cm}^{-1}\right): v(C=\mathrm{N}) 1593 ; v(\mathrm{P}-\mathrm{F})$ 845. Anal. Calcd for $\mathrm{C}_{28} \mathrm{H}_{32} \mathrm{~N}_{6} \mathrm{O}_{2} \mathrm{PF}_{6} \mathrm{Ru}: \mathrm{C}, 46.03 ; \mathrm{H}, 4.41 ; \mathrm{N}, 11.50$. Found: $\mathrm{C}, 45.87$; $\mathrm{H}, \quad 4.56 ; \quad \mathrm{N}, \quad 11.37$. UV-vis $\left(\mathrm{CH}_{3} \mathrm{CN}\right): \lambda_{\max } \quad[\mathrm{nm}] \quad(\varepsilon$ $\left.\left[\mathrm{mol}^{-1} \mathrm{dm}^{3} \mathrm{~cm}^{-1}\right]\right) 680$ (4955), 497 (1940), 388 (17 540), 343sh (12 890), 234sh (44 590), 214 (49 270). ESI-MS: $\mathrm{m} / z=586\left(\mathrm{M}^{+}\right)$ $\mu_{\mathrm{eff}}=1.96 \mu_{\mathrm{B}}$.

$\left[\mathbf{R u}^{\mathrm{III}}\left(\mathbf{L}^{1}\right)(\mathbf{p y})_{2}\right]\left(\mathbf{P F}_{\mathbf{6}}\right)$ (4). The green solid was prepared by a procedure similar to that for $\mathbf{3}$ using pyridine. Yield: (76\%). IR $\left(\mathrm{KBr}, \mathrm{cm}^{-1}\right): v(C=\mathrm{N})$ 1597; $v(\mathrm{P}-\mathrm{F})$ 845. Anal. Calcd for

(40) Thornback, J. R.; Wilkinson, G. J. Chem. Soc., Dalton Trans. 1978, $110-115$.

(41) Man, W. L.; Tang, T. M.; Wong, T. W.; Lau, T. C.; Peng, S. M.; Wong, W. T. J. Am. Chem. Soc. 2004, 126, 478-479.

(42) Griffith, W. P.; Pawson, D. J. Chem. Soc., Dalton Trans. 1973, 13151320.

(43) Wong, C. Y.; Man, W. L.; Wong, C.; Kwong, H. L.; Wong, W. Y.; Lau, T. C. Organometallics 2008, 27, 324-326. 


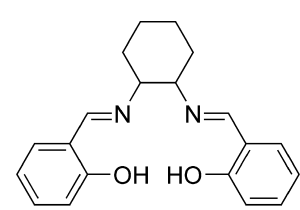

$\mathrm{H}_{2} \mathrm{~L}^{1}$

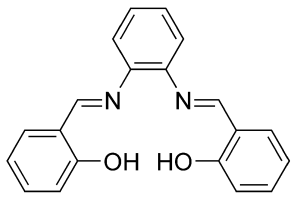

$\mathrm{H}_{2} \mathrm{~L}^{2}$

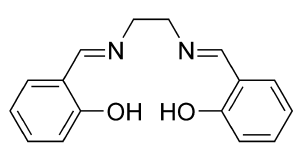

$\mathrm{H}_{2} \mathrm{~L}^{3}$

Figure 1. Structures of salen ligands.

Table 1. Crystal Data and Structure Refinement Details for 6, 9, 11, and $\mathbf{1 2}$

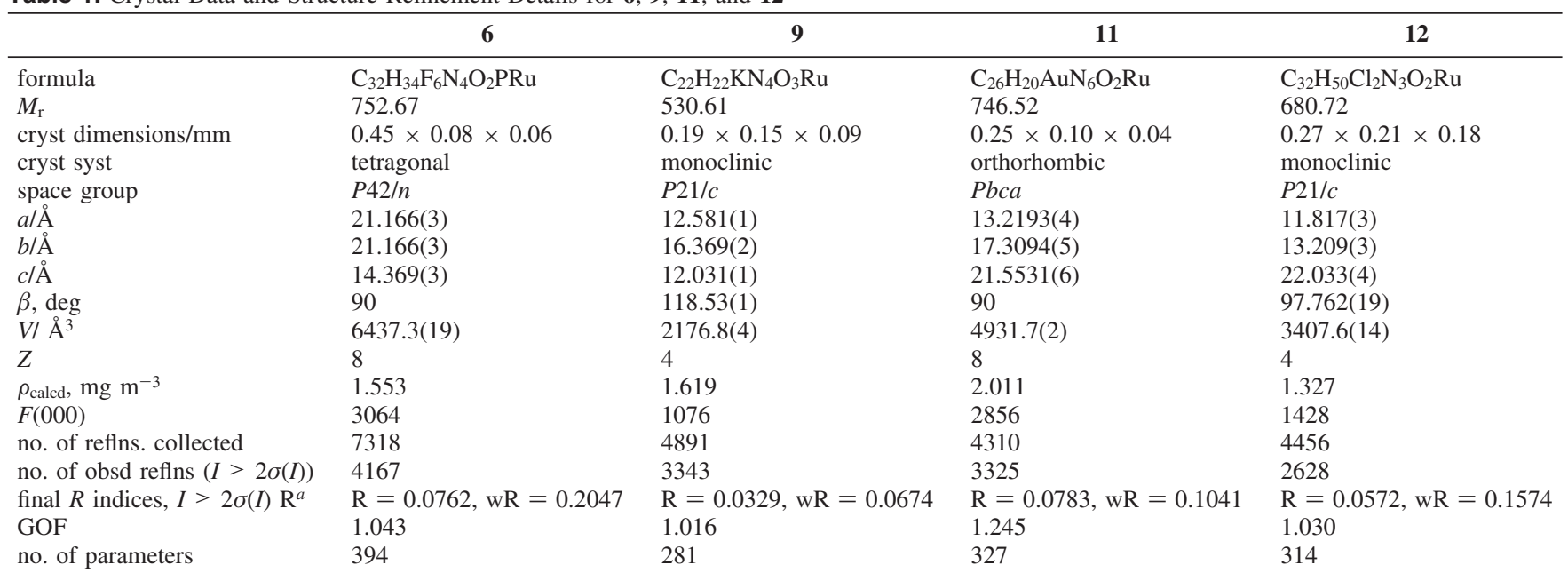

$\mathrm{C}_{30} \mathrm{H}_{30} \mathrm{~N}_{4} \mathrm{O}_{2} \mathrm{PF}_{6} \mathrm{Ru}: \mathrm{C}, 49.73 ; \mathrm{H}, 4.17 ; \mathrm{N}, 7.73$. Found: C, 49.60; $\mathrm{H}, 4.19 ; \mathrm{N}, 7.64$. UV-vis $\left(\mathrm{CH}_{3} \mathrm{CN}\right): \lambda_{\max }[\mathrm{nm}]\left(\varepsilon\left[\mathrm{mol}^{-1} \mathrm{dm}^{3} \mathrm{~cm}^{-1}\right]\right)$ 723 (4650), 373 (16 490), 235 (43 650), 215 (41 510). ESI-MS: $m / z=580\left(\mathrm{M}^{+}\right) . \mu_{\text {eff }}=1.90 \mu_{\mathrm{B}}$.

$\left[\mathbf{R u}{ }^{\mathrm{III}}\left(\mathbf{L}^{1}\right)\left(\mathbf{M e}_{2} \mathbf{S O}\right)_{2}\right]\left(\mathbf{P F}_{6}\right)(\mathbf{5})$. The green solid was prepared by a procedure similar to that for 3 using $\mathrm{Me}_{2} \mathrm{SO}$. Yield: (66\%). IR $\left(\mathrm{KBr}, \mathrm{cm}^{-1}\right): v(C=\mathrm{N}) 1599 ; v(\mathrm{~S}=\mathrm{O}) 1017 ; v(\mathrm{P}-\mathrm{F})$ 840. Anal. Calcd for $\mathrm{C}_{24} \mathrm{H}_{32} \mathrm{~N}_{2} \mathrm{O}_{4} \mathrm{~S}_{2} \mathrm{PF}_{6} \mathrm{Ru}$ : C, 39.89; $\mathrm{H}, 4.46 ; \mathrm{N}, 3.88$. Found: $\mathrm{C}, 40.10 ; \mathrm{H}, 4.41 ; \mathrm{N}, 4.10$. UV-vis $\left(\mathrm{CH}_{3} \mathrm{CN}\right): \lambda_{\max }[\mathrm{nm}](\varepsilon$ $\left.\left[\mathrm{mol}^{-1} \mathrm{dm}^{3} \mathrm{~cm}^{-1}\right]\right) 734$ (5015), 481 (1660), 332 (16 960), 232 (43 780). ESI-MS: $m / z=578\left(\mathrm{M}^{+}\right) . \mu_{\mathrm{eff}}=2.07 \mu_{\mathrm{B}}$.

$\left[\mathbf{R u}{ }^{\mathrm{III}}\left(\mathbf{L}^{\mathbf{1}}\right)\left(\mathbf{N H}_{\mathbf{2}} \mathbf{P h}\right)_{\mathbf{2}}\right]\left(\mathbf{P F}_{\mathbf{6}}\right) \mathbf{( 6 )}$. Aniline (0.32 $\left.\mathrm{mL}, 3.52 \mathrm{mmol}\right)$ was added to a solution of 2 (100 $\mathrm{mg}, 0.16 \mathrm{mmol})$ in ethanol (15 $\mathrm{mL})$. The green solution was refluxed for $1.5 \mathrm{~h}$ and then concentrated to ca. $1 \mathrm{~mL}$. Slow addition of diethyl ether gave a green precipitate, which was recrystallized from acetone/diethyl ether. Yield: $51 \%$. Single crystals suitable for X-ray crystallography were obtained by slow diffusion of diethyl ether into an acetone solution of the compound. IR $\left(\mathrm{KBr}, \mathrm{cm}^{-1}\right): v(\mathrm{~N}-\mathrm{H}) 3311,3278$; $v(C=\mathrm{N}) 1602 ; v(\mathrm{P}-\mathrm{F})$ 835. Anal. Calcd for $\mathrm{C}_{32} \mathrm{H}_{34} \mathrm{~N}_{4} \mathrm{O}_{2} \mathrm{PF}_{6} \mathrm{Ru}$ : C, 51.06; H, 4.55; N, 7.44. Found: C, 51.23; H, 4.62; N, 7.21. $\mathrm{UV}-\mathrm{vis}\left(\mathrm{CH}_{3} \mathrm{CN}\right): \lambda_{\max }[\mathrm{nm}]\left(\varepsilon\left[\mathrm{mol}^{-1} \mathrm{dm}^{3} \mathrm{~cm}^{-1}\right]\right) 705$ (4880), 492 (1870), 363 (14 530), 346 (14 150), 233 (47 960), 202 (56 570). ESI-MS: $m / z=608\left(\mathrm{M}^{+}\right) \cdot \mu_{\mathrm{eff}}=1.86 \mu_{\mathrm{B}}$.

$\left[\mathbf{R} \mathbf{u}^{\mathrm{III}}\left(\mathbf{L}^{1}\right)\left(\mathbf{N H}_{2}{ }^{t} \mathbf{B u}\right)_{2}\right]\left(\mathbf{P F}_{6}\right)(\mathbf{7})$. The green solid was prepared by a procedure similar to that for $\mathbf{6}$ using tert-butylamine $(0.34 \mathrm{~mL}$, 3.25 mmol). Yield: (40\%). IR ( $\left.\mathrm{KBr}, \mathrm{cm}^{-1}\right): v(\mathrm{~N}-\mathrm{H}) 3300,3244$; $v(C=\mathrm{N}) 1600 ; v(\mathrm{P}-\mathrm{F})$ 849. Anal. Calcd. for $\mathrm{C}_{28} \mathrm{H}_{42} \mathrm{~N}_{4} \mathrm{O}_{2} \mathrm{PF}_{6} \mathrm{Ru}$ : C, 47.19; H, 5.94; N, 7.86. Found: C, 47.15; H, 5.75; N, 7.81. $\mathrm{UV}-\mathrm{vis}\left(\mathrm{CH}_{3} \mathrm{CN}\right): \lambda_{\max }[\mathrm{nm}]\left(\varepsilon\left[\mathrm{mol}^{-1} \mathrm{dm}^{3} \mathrm{~cm}^{-1}\right]\right) 670$ (5920), 497 (2510), 387 (20 100), 366 (17 260), 234 (42 970), 217 (44 440) ESIMS: $m / z=568\left(\mathrm{M}^{+}\right) \cdot \mu_{\mathrm{eff}}=2.02 \mu_{\mathrm{B}}$.

$\left[\mathbf{N}^{\mathbf{n}} \mathbf{B u}_{4}\right]\left[\mathbf{R u}^{\mathrm{III}}\left(\mathbf{L}^{\mathbf{1}}\right) \mathbf{C l}_{2}\right](\mathbf{8}) .\left[\mathrm{N}^{n} \mathrm{Bu} \mathrm{u}_{4} \mathrm{Cl}\right](0.55 \mathrm{~g}, 2.0 \mathrm{mmol})$ was added to an orange solution of $\mathbf{1}(120 \mathrm{mg}, 0.2 \mathrm{mmol})$ in acetone $(20 \mathrm{~mL})$ and refluxed for 1 day. The green solution was then concentrated to ca. $1 \mathrm{~mL}$. Addition of diethyl ether $(20 \mathrm{~mL})$ gave a green solid, which was recrystallized from $\mathrm{CH}_{2} \mathrm{Cl}_{2} / \mathrm{Et}_{2} \mathrm{O}$. Yield:
(70\%). IR $\left(\mathrm{KBr}, \mathrm{cm}^{-1}\right): \quad v(C=\mathrm{N})$ 1597. Anal. Calcd for $\mathrm{C}_{36} \mathrm{H}_{56} \mathrm{~N}_{3} \mathrm{O}_{2} \mathrm{Cl}_{2} \mathrm{Ru} \cdot \mathrm{CH}_{2} \mathrm{Cl}_{2}$ : C, 54.48; H, 7.19; N, 5.08. Found: $\mathrm{C}$, 54.54; H, 6.91; N, 4.93. UV/vis $\left(\mathrm{CH}_{3} \mathrm{CN}\right): \lambda_{\max }[\mathrm{nm}](\varepsilon$ $\left.\left[\mathrm{mol}^{-1} \mathrm{dm}^{3} \mathrm{~cm}^{-1}\right]\right) 645$ (5120), 514 (2395), 415 (21 930), 346 (11 850), 292 (8110), 238 (47 600), 221 (47 360). ESI-MS: $\mathrm{m} / \mathrm{z}=$ $494\left(\mathrm{M}^{-}\right) . \mu_{\mathrm{eff}}=2.06 \mu_{\mathrm{B}}$.

$\mathbf{K}\left[\mathbf{R} \mathbf{u}^{\text {III }}\left(\mathbf{L}^{\mathbf{1}}\right)(\mathbf{C N})_{2}\right] \cdot \mathbf{H}_{\mathbf{2}} \mathbf{O}(\mathbf{9}) . \mathrm{KCN}(130 \mathrm{mg}, 2 \mathrm{mmol})$ was added to an orange solution of $\mathbf{1}(123 \mathrm{mg}, 0.2 \mathrm{mmol})$ in methanol (50 $\mathrm{mL}$ ), and the mixture was stirred for $3 \mathrm{~h}$ at room temperature. Slow evaporation of the resulting green solution afforded dark green single crystals suitable for X-ray crystallography. Yield: $52 \%$. IR $\left(\mathrm{KBr}, \mathrm{cm}^{-1}\right): v(\mathrm{C} \equiv \mathrm{N}) 2090 ; v(C=\mathrm{N})$ 1598. Anal. Calcd for $\mathrm{C}_{22} \mathrm{H}_{22} \mathrm{~N}_{4} \mathrm{O}_{3} \mathrm{KRu}$ : C, 49.80; H, 4.18; N, 10.56. Found: C, 49.95; H, 4.33; N, 10.37. ESI-MS: $m / z=474\left(\mathrm{M}^{-}\right) . \mu_{\mathrm{eff}}=1.97 \mu_{\mathrm{B}}$.

$\left[\mathbf{R} \mathbf{u}^{\mathbf{V I}}(\mathbf{N})\left(\mathbf{L}^{2}\right) \mathbf{C l}\right]$ (10a). 2,6-dimethylpyridine $(0.2 \mathrm{~mL})$ was added dropwise to a solution of $\left[\mathrm{N}^{n} \mathrm{Bu}_{4}\right]\left[\mathrm{Ru}^{\mathrm{VI}}(\mathrm{N}) \mathrm{Cl}_{4}\right](0.2 \mathrm{~g}, 0.4 \mathrm{mmol})$ and $\mathrm{H}_{2} \mathrm{~L}^{2}$ (0.13 g, $\left.0.4 \mathrm{mmol}\right)$ in $\mathrm{CH}_{2} \mathrm{Cl}_{2}(20 \mathrm{~mL})$. The deep-brown solution was stirred at room temperature for $0.5 \mathrm{~h}$. The resulting brown solid was collected, washed with $\mathrm{CH}_{2} \mathrm{Cl}_{2}$, diethyl ether and then air-dried. Yield: (80\%). ${ }^{1} \mathrm{H}$ NMR (300 MHz, $\left.\mathrm{CD}_{3} \mathrm{OD}\right): \delta 7.18$ (t, 2H), 7.48 (d, 2H), $7.74(\mathrm{~m}, 2 \mathrm{H}), 7.85(\mathrm{~m}, 2 \mathrm{H}), 8.01(\mathrm{~d}, 2 \mathrm{H})$, $8.49(\mathrm{~m}, 2 \mathrm{H}), 9.89(\mathrm{~s}, 2 \mathrm{H}) . \mathrm{IR}\left(\mathrm{KBr}, \mathrm{cm}^{-1}\right): v\left(\mathrm{Ru} \equiv{ }^{14} \mathrm{~N}\right) 1036$; $v\left(\mathrm{Ru} \equiv{ }^{15} \mathrm{~N}\right)$ 1006. Anal. Calcd for $\mathrm{C}_{20} \mathrm{H}_{14} \mathrm{~N}_{3} \mathrm{O}_{2} \mathrm{ClRu}$ : C, 51.67; $\mathrm{H}$, 3.01; N, 9.04. Found: C, 51.41; H, 3.12; N, 8.89. ESI-MS: $\mathrm{m} / \mathrm{z}=$ $430\left(\mathrm{M}^{+}-\mathrm{Cl}\right)$. Conductivity in $\mathrm{CH}_{3} \mathrm{OH}: \Lambda=53 \Omega^{-1} \mathrm{~cm}^{2} \mathrm{~mol}^{-1}$.

$\left[\mathbf{R u}{ }^{\mathrm{VI}}(\mathbf{N})\left(\mathbf{L}^{2}\right)(\mathbf{M e O H})\right]\left(\mathbf{C l O}_{4}\right)$ (10b). 10a (0.186 g, $\left.0.4 \mathrm{mmol}\right)$ was dissolved in methanol $(30 \mathrm{~mL})$ and silver $p$-toluenesulfonate (AgOTs) (0.112 g, $0.4 \mathrm{mmol}$ ) was added. The mixture was stirred at room temperature for $0.5 \mathrm{~h}$ and then filtered to remove $\mathrm{AgCl}$. To the concentrated filtrate excess $\mathrm{LiClO}_{4}(0.1 \mathrm{~g}, 0.9 \mathrm{mmol})$ was added and the deep reddish-brown solution was cooled in ice. The resulting brick-red microcrystalline solid was collected, washed with methanol, and then diethyl ether. Yield: (65\%). ${ }^{1} \mathrm{H}$ NMR (300 MHz, $\left.\mathrm{CD}_{3} \mathrm{OD}\right): \delta 7.17(\mathrm{t}, 2 \mathrm{H}), 7.48(\mathrm{~d}, 2 \mathrm{H}), 7.73(\mathrm{~m}, 2 \mathrm{H}), 7.86(\mathrm{~m}, 2 \mathrm{H})$, $8.03(\mathrm{~d}, 2 \mathrm{H}), 8.49(\mathrm{~m}, 2 \mathrm{H}), 9.89(\mathrm{~s}, 2 \mathrm{H})$. Anal. Calcd for 
Table 2. UV-vis $\left(\mathrm{CH}_{3} \mathrm{CN}\right)$ and Electrochemical Data for $\mathbf{3}-\mathbf{8}$ and $\mathbf{1 2}$

\begin{tabular}{|c|c|c|c|}
\hline \multirow[b]{2}{*}{ complex } & \multirow[b]{2}{*}{$\lambda_{\max }\left(\mathrm{nm}\left(\epsilon, \mathrm{M}-1, \mathrm{~cm}^{-1}\right)\right)$} & \multicolumn{2}{|c|}{$E_{1 / 2}\left(\text { Volts vs } \mathrm{Cp}_{2} \mathrm{Fe}^{+/ 0}\right)^{a}$} \\
\hline & & $\mathrm{Ru}^{\mathrm{IV} / I I I}$ & $\mathrm{Ru}^{\mathrm{III} / \mathrm{II}}$ \\
\hline 3 & $\begin{array}{l}680 \text { (4955), } 497 \text { (1940), } 388 \text { (17 540), 343sh (12 890), 234sh (44 590), } \\
214 \text { (49 270) }\end{array}$ & 0.54 & -0.89 \\
\hline 5 & $734(5015), 481(1660), 332(16960), 232(43780)$ & $0.89^{b}$ & -0.30 \\
\hline 6 & $\begin{array}{l}705 \text { (4880), } 492 \text { (1870), } 363 \text { (14 530), } 346 \text { (14 150), } 233 \text { (47 960), } 202 \\
\quad(56570)\end{array}$ & $0.58^{b}$ & -0.86 \\
\hline 8 & $\begin{array}{l}645(5120), 514(2395), 415 \text { (21 930), } 346 \text { (11 850), } 292 \text { (8110), } 238 \\
\quad(47600), 221(47360)\end{array}$ & 0.10 & $-1.50^{b}$ \\
\hline 12 & 647 (2360), 515 (1000), 415 (10 240), 347 (5880), 237 (24 400), 222 & 0.16 & $-1.39^{b}$ \\
\hline
\end{tabular}
(25000)

${ }^{a}$ Glassy carbon working electrode, platinum counter electrode, $\mathrm{Ag} / \mathrm{AgNO}_{3}$ reference electrode, $0.1 \mathrm{M}\left[\mathrm{N}^{n} \mathrm{Bu}_{4}\right] \mathrm{PF}_{6}$ in $\mathrm{CH}_{3} \mathrm{CN}_{\text {as supporting electrode. }}$ Ferrocene was added as internal standard. ${ }^{b}$ Irreversible.

$\mathrm{C}_{21} \mathrm{H}_{18} \mathrm{~N}_{3} \mathrm{O}_{7} \mathrm{ClRu}: \mathrm{C}, 44.96 ; \mathrm{H}, 3.21 ; \mathrm{N}, 7.49$. Found: C, 44.72; H, $3.11 ; \mathrm{N}, 7.36$. ESI-MS: $\mathrm{m} / \mathrm{z}=430\left(\mathrm{M}^{+}\right)$. Conductivity in $\mathrm{CH}_{3} \mathrm{OH}$ : $\Lambda=87 \Omega^{-1} \mathrm{~cm}^{2} \mathrm{~mol}^{-1}$.

Caution! Perchlorate salts are potentially explosive. Although we have not encountered any explosions so far, the amount of perchlorate salts used should be less than $100 \mathrm{mg}$ each time.

$\left[\mathbf{R u}^{\mathrm{III}}\left(\mathbf{L}^{2}\right)\left(\mathrm{NCCH}_{3}\right)_{2}\right]\left[\mathrm{Au}^{\mathrm{I}}(\mathbf{C N})_{2}\right]$ (11). A mixture of 10a and $\mathrm{K}\left[\mathrm{Au}^{\mathrm{I}}(\mathrm{CN})_{2}\right]$ in $\mathrm{CH}_{3} \mathrm{OH} / \mathrm{H}_{2} \mathrm{O}(20 \mathrm{~mL}, 4: 1 \mathrm{v} / \mathrm{v})$ was stirred at 30 ${ }^{\circ} \mathrm{C}$ for $1 \mathrm{~h}$. The deep-brown solution was concentrated to give a light-brown solid, which was recrystallized by slow evaporation of a solution in $\mathrm{CH}_{3} \mathrm{CN} / \mathrm{H}_{2} \mathrm{O}(2: 1 \mathrm{v} / \mathrm{v})$. Yield $(30 \%)$. IR $(\mathrm{KBr}$, $\left.\mathrm{cm}^{-1}\right): v(\mathrm{C} \equiv \mathrm{N}) 2140 ; v(C=\mathrm{N})$ 1597. Anal. Calcd for $\mathrm{C}_{26} \mathrm{H}_{22^{-}}$ $\mathrm{N}_{6} \mathrm{O}_{2} \mathrm{AuRu}$ : C, 41.82; H, 2.68; N, 11.26. Found: C, 42.02; H, 2.80; N, 11.03. ESI-MS: $m / z=498\left(\mathrm{M}^{+}\right)$.

$\left[\mathbf{N}^{n} \mathbf{B u}_{4}\right]\left[\mathbf{R u}^{\mathrm{III}}\left(\mathbf{L}^{3}\right) \mathbf{C l}_{\mathbf{2}}\right] \quad(\mathbf{1 2}) .\left[\mathrm{N}^{n} \mathrm{Bu}_{4}\right]\left[\mathrm{Ru}^{\mathrm{VI}}(\mathrm{N}) \mathrm{Cl}_{4}\right](0.2 \mathrm{~g}, 0.4$ mmol) was dissolved in THF $(20 \mathrm{~mL})$ and $\mathrm{H}_{2} \mathrm{~L}^{3}(0.108 \mathrm{~g}, 0.4 \mathrm{mmol})$ was added. The solution was stirred at room temperature for $16 \mathrm{~h}$ and then evaporated to dryness. The resulting green residue was dissolved in $\mathrm{CH}_{2} \mathrm{Cl}_{2}$ and purified by column chromatography (neutral alumina) with $\mathrm{CH}_{2} \mathrm{Cl}_{2}$ as eluant. Dark-green crystals were obtained by diffusion of diethyl ether into a concentrated $\mathrm{CH}_{2} \mathrm{Cl}_{2}$ solution of the solid. Yield: (45\%). IR $\left(\mathrm{KBr}, \mathrm{cm}^{-1}\right): v(C=\mathrm{N}) 1601$. Anal. Calcd for $\mathrm{C}_{32} \mathrm{H}_{50} \mathrm{~N}_{3} \mathrm{O}_{2} \mathrm{Cl}_{2} \mathrm{Ru} \cdot \mathrm{CH}_{2} \mathrm{Cl}_{2}$ : C, 51.77; $\mathrm{H}, 6.85 ; \mathrm{N}$, 5.49. Found: C, 51.92; H, 6.63; N, 5.49. ESI-MS: $m / z=438\left(\mathrm{M}^{-}\right)$. Conductivity in $\mathrm{CH}_{3} \mathrm{CN}: \Lambda=111 \Omega^{-1} \mathrm{~cm}^{2} \mathrm{~mol}^{-1} \cdot \mu_{\mathrm{eff}}=1.79 \mu_{\mathrm{B}}$.

Kinetics. Kinetic experiments were done using either a PerkinElmer Lamda 19 or a Hewlett-Packard 8452A diode-array UV-vis spectrophotometer. The ionic strengths of the solutions were maintained with ${ }^{n} \mathrm{Bu}_{4} \mathrm{NPF}_{6}$. The concentrations of py $\left(4 \times 10^{-2}\right.$ to $3.0 \mathrm{M})$ were at least in 10 -fold excess of that of $1\left(1 \times 10^{-5}\right.$ to 5 $\left.\times 10^{-5} \mathrm{M}\right)$. The reaction progress was monitored by observing the absorbance changes at $724 \mathrm{~nm}$. Pseudo-first-order rate constants, $k_{\mathrm{obs}}$, were obtained by nonlinear least-squares fits of $A_{\mathrm{t}}$ versus time $t$ according to the equation $A_{\mathrm{t}}=A_{\infty}+\left(A_{0}-A_{\infty}\right) \exp \left(-k_{\text {obs }} t\right)$, where $A_{0}$ and $A_{\infty}$ are the initial and final absorbances, respectively.

X-ray Crystallography. All measurements of 6, 9, 11, and 12 were made on either a Bruker Smart 1000 CCD or a Rigaku AFC7R diffractometer with graphite-monochromated Mo $\mathrm{K} \alpha$ radiation $(\lambda$ $=0.71073 \AA$ ) in the $\omega$-scan mode. Details of the intensity data collection and crystal data are given in Table 1 . The data were corrected for Lorentz and polarization effects. Absorption corrections by the $\Psi$-scan method or an approximation by interimage scaling were applied. The structures were resolved by direct methods ${ }^{44}$ and expanded using Fourier techniques. ${ }^{45}$ All calcula-

(44) Altomare, A.; Cascarano, G.; Giacovazzo, C.; Guagliardi, A.; Burla, M.; Polidori, G.; Camalli, M. J. Appl. Crystallogr. 1994, 27, 435.

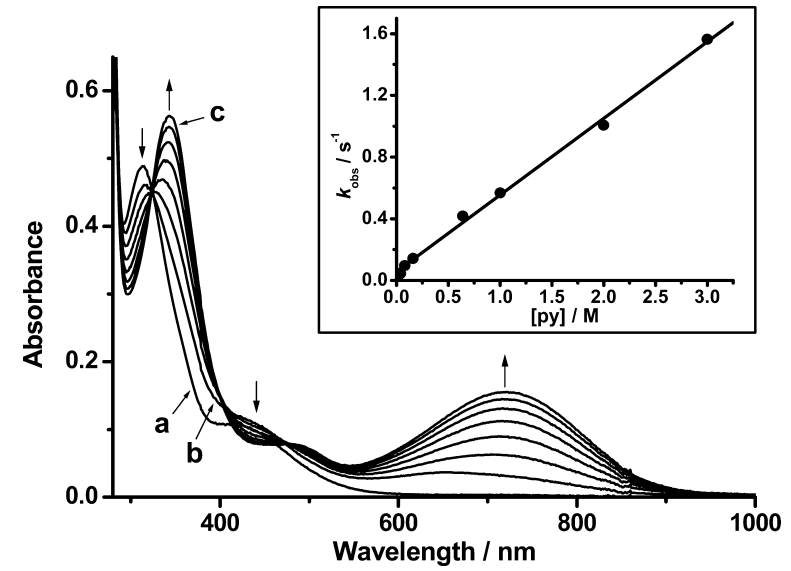

Figure 2. Spectral changes at $180 \mathrm{~s}$ intervals for the reaction of $\mathbf{1}(4 \times$ $\left.10^{-5} \mathrm{M}\right)$ with py $(0.01 \mathrm{M})$ in $\mathrm{CH}_{3} \mathrm{CN}$ at $298.0 \mathrm{~K}$ and $I=0.05 \mathrm{M}(a=$ initial, $b=$ immediately after mixing using a two-compartment cell, $c$ $=$ final). Inset shows the plot of $k_{\text {obs }}$ vs [py] for the second step (b $\rightarrow$ c) [slope $=(4.96 \pm 0.12) \times 10^{-1} ; \mathrm{y}$ intercept $=(5.81 \pm 0.17) \times 10^{-2}$; $r=0.9986]$.

tions were performed using the Crystal Structure or TeXsan crystallographic software package from Molecular Structure Corporation. ${ }^{46}$

\section{Results and Discussion}

Ligand-Induced $N \cdots N$ coupling of $\left[R^{V I}(N)\left(L^{1}\right)\right.$ $(\mathrm{MeOH})]^{+}(\mathbf{1})$. A solution of 1 in $\mathrm{CH}_{3} \mathrm{CN}\left(4.0 \times 10^{-5} \mathrm{M}\right)$ is stable for at least $24 \mathrm{~h}$ at room temperature. However, a very rapid spectral change occurs when excess pyridine (1.0 $\times 10^{-2} \mathrm{M}$ ) is added; this is followed by a much slower step with isosbestic points at 323, 397, and $478 \mathrm{~nm}$. The final spectrum is consistent with the quantitative formation of $\left[\mathrm{Ru}^{\mathrm{III}}\left(\mathrm{L}^{1}\right)(\mathrm{py})_{2}\right]^{+}$(4). The formation of $\mathbf{4}$ follows clean pseudo-first-order kinetics; the pseudo-first-order rate constant, $k_{\mathrm{obs}}$, is independent of [1] $\left(1 \times 10^{-5}\right.$ to $\left.5 \times 10^{-5} \mathrm{M}\right)$ but depends linearly on [py]. At $298.0 \mathrm{~K}$ and $I=0.05 \mathrm{M}$, the second-order rate constant, $k_{2}$, is $(4.96 \pm 0.12) \times 10^{-1}$ $\mathrm{M}^{-1} \mathrm{~s}^{-1}$ (Figure 2).

When higher concentrations of $\mathrm{Ru}^{\mathrm{VI}}$ are used $\left(>2 \times 10^{-2}\right.$ $\mathrm{M})$, gas bubbles of $\mathrm{N}_{2}$ (GC/MS) can be observed upon adding

(45) DIRDIF 99, Beurskens, P. T.; Admiraal, G.; Beurskens, G.; Bosman, W. P.; de Gelder, R.; Israel, R.; Smits, J. M. M. The DIRDIF-99 program system; Technical Report of the Crystallography Laboratory, University of Nijmegen: The Netherlands, 1999. 
excess pyridine. Using ${ }^{15} \mathrm{~N}$ labeling, it is shown that both nitrogen atoms in $\mathrm{N}_{2}$ come from $\mathrm{Ru}^{\mathrm{VI}} \equiv \mathrm{N}$. ${ }^{41}$ These results are consistent with the reaction scheme shown in eqs 6-9. The observed rapid first step is probably due to eqs 6 and 7 , whereas the second step can be accounted for by eqs 8 and 9, with 8 being the rate-limiting step.

$\left[\mathrm{Ru}^{\mathrm{VI}}(\mathrm{N})\left(\mathrm{L}^{1}\right)(\mathrm{NCCH})_{3}\right]^{+}+\mathrm{py} \rightleftharpoons\left[\mathrm{Ru}^{\mathrm{VI}}(\mathrm{N})\left(\mathrm{L}^{1}\right)(\mathrm{py})\right]^{+}+$ $\mathrm{CH}_{3} \mathrm{CN}(6)$

$$
\begin{aligned}
& 2\left[\mathrm{Ru}^{\mathrm{VI}}(\mathrm{N})\left(\mathrm{L}^{1}\right)(\mathrm{py})\right]+ \\
& \rightarrow\left[(\text { py })\left(\mathrm{L}^{1}\right) \mathrm{Ru}^{\mathrm{III}} \mathrm{N} \equiv \mathrm{NRu}^{\mathrm{III}}\left(\mathrm{L}^{1}\right)(\mathrm{py})\right]^{2+} \\
& {\left[\left(\text { py) }\left(\mathrm{L}^{1}\right) \mathrm{Ru}{ }^{\mathrm{III}} \mathrm{N} \equiv \mathrm{NRu}{ }^{\mathrm{III}}\left(\mathrm{L}^{1}\right)(\mathrm{py})\right]^{2+}+\right.} \\
& \text { py } \rightarrow\left[R u^{\text {III }}\left(\mathrm{L}^{1}\right)(\text { py })_{2}\right]^{+}+\left[\mathrm{Ru}^{\mathrm{III}}\left(\mathrm{L}^{1}\right)(\mathrm{py})\left(\mathrm{N}_{2}\right)\right]^{+} \text {(slow) }(8) \\
& {\left[\mathrm{Ru}^{\mathrm{III}}\left(\mathrm{L}^{1}\right)(\mathrm{py})\left(\mathrm{N}_{2}\right)\right]^{+}+\mathrm{py} \rightarrow\left[\mathrm{Ru}^{\mathrm{III}}\left(\mathrm{L}^{1}\right)(\mathrm{py})_{2}\right]^{+}+\mathrm{N}_{2}}
\end{aligned}
$$

In the pyridine-activated aziridination of alkenes by $\mathbf{1}$, there is kinetic evidence for the equilibrium described in eq 6, with $K=15.6 \mathrm{M}^{-1}$ at $298 \mathrm{~K}^{47}$ The $\left[\mathrm{Ru}^{\mathrm{VI}}(\mathrm{N})\left(\mathrm{L}^{1}\right)(\mathrm{py})\right]^{+}$ species undergoes rapid $\mathrm{N} \cdots \mathrm{N}$ coupling to produce a $\mu-\mathrm{N}_{2}$ $\mathrm{Ru}^{\mathrm{III}}$ species (eq 7). Rapid $\mathrm{N} \cdots \mathrm{N}$ coupling of $\left[\mathrm{Os}^{\mathrm{V}}\left(\mathrm{NH}_{3}\right)_{4^{-}}\right.$ $(\mathrm{N})]^{2+}$, generated from photolysis of $\left[\mathrm{Os}^{\mathrm{VI}}\left(\mathrm{NH}_{3}\right)_{4}(\mathrm{~N})\right]^{3+}$ in the presence of an electron donor, has been observed with $k$ $=3.75 \times 10^{5} \mathrm{M}^{-1} \mathrm{~s}^{-1}{ }^{48}$ Because there should not be significant $\pi$ back bonding between $\mathrm{N}_{2}$ and $\mathrm{Ru}^{\mathrm{III}}$, loss of bridging or terminal $\mathrm{N}_{2}$ from $\mathrm{Ru}^{\mathrm{III}} \mathrm{L}^{1}$ is expected to be facile (eqs 8 and 9). The y intercept of $5.81 \times 10^{-2} \mathrm{~s}^{-1}$ in the plot of $k_{\text {obs }}$ versus [py] is probably due to a minor solvolysis pathway shown in eq 10 .

$$
\begin{aligned}
& {\left[(\text { py })\left(\mathrm{L}^{1}\right) \mathrm{Ru}^{\mathrm{III}} \mathrm{N} \equiv \mathrm{N}-\mathrm{Ru}^{\mathrm{III}}\left(\mathrm{L}^{1}\right)(\mathrm{py})\right]^{2+}+} \\
& \mathrm{CH}_{3} \mathrm{CN} \rightarrow\left[\mathrm{Ru}^{\mathrm{III}}\left(\mathrm{L}^{1}\right)(\mathrm{py})\left(\mathrm{NCCH}_{3}\right)\right]^{+}+\left[\mathrm{Ru}^{\mathrm{III}}\left(\mathrm{L}^{1}\right)(\mathrm{py})\left(\mathrm{N}_{2}\right)\right]^{+}
\end{aligned}
$$

The assumption that eq 8 is the rate-limiting step is valid if the coupling rate constant of eq 7 is similar to (or faster than) that of $\left[\mathrm{Os}^{\mathrm{V}}\left(\mathrm{NH}_{3}\right)_{4}(\mathrm{~N})\right]^{2+}\left(4 \times 10^{5} \mathrm{M}^{-1} \mathrm{~s}^{-1}\right)$. On the basis of the concentrations of $\mathbf{1}\left(4 \times 10^{-5} \mathrm{M}\right)$ and py $(0.01$ $\mathrm{M})$ in the experiment, and the equilibrium constant of eq 6 $\left(K=15.6 \mathrm{M}^{-1}\right)$, the equilibrium concentration of the adduct $\left[\mathrm{Ru}^{\mathrm{VI}}(\mathrm{N})\left(\mathrm{L}^{1}\right)(\mathrm{py})\right]^{+}$is $6.2 \times 10^{-6} \mathrm{M}$. If the coupling rate constant of eq 7 is $4 \times 10^{5} \mathrm{M}^{-1} \mathrm{~s}^{-1}$, then the coupling rate in eq 7 is $1.6 \times 10^{-5} \mathrm{M} \mathrm{s}^{-1}$ at $298 \mathrm{~K}$, which is around two orders of magnetude faster than the observed rate $2 \times 10^{-7}$ $\mathrm{M} \mathrm{s}^{-1}$ (which is assumed to be that of eq 8).

Attempts to detect the proposed $\left[(p y)\left(\mathrm{L}^{1}\right) \mathrm{Ru}^{\mathrm{III}} \mathrm{N} \equiv \mathrm{NRu}^{\mathrm{III}}\left(\mathrm{L}^{1}\right)\right.$ $(\mathrm{py})]^{2+}$ intermediate by ESI-MS were unsuccessful. The mass spectrum $(+$ ve mode $)$ of $(\mathbf{1})\left(4.0 \times 10^{-5} \mathrm{M}\right)$ and pyridine $(0.01 \mathrm{M})$ in $\mathrm{CH}_{3} \mathrm{CN}$ collected 5 min after mixing shows only

(46) CrystalStructure, Single Crystal Structure Analysis Software, version 3.5.1; Rigaku/MSC Corporation: The Woodlands, Texas, USA, Rigaku, Akishima, Tokyo, Japan, 2003; Watkin, D. J. Prout. C. K., Carruthers. J. R., Betteridge, P. W. Crystals, Chemical Crystallography Lab: Oxford, UK, 1996; issue 10.

(47) Man, W. L.; Lam, W. W. Y.; Yiu, S. M.; Lau, T. C.; Peng, S. M. J. Am. Chem. Soc. 2004, 126, 15336-15337.

(48) Lam, H. W.; Che, C. M.; Wong, K. Y. J. Chem. Soc., Dalton Trans. 1992, 1411-1416. peaks due to $[\mathrm{Ru}(\mathrm{L})]^{+}(\mathrm{m} / \mathrm{z}=422.1),[\operatorname{Ru}(\mathrm{N})(\mathrm{L})]^{+}(\mathrm{m} / \mathrm{z}=$ 436.2), $\left[\mathrm{Ru}(\mathrm{L})\left(\mathrm{NCCH}_{3}\right)\right]^{+}(m / z=463.2),\left[\mathrm{Ru}(\mathrm{L})\left(\mathrm{NCCH}_{3}\right)_{2}\right]^{+}$ $(m / z=504.3)$ and $\left[\mathrm{Ru}(\mathrm{L})(\mathrm{py})\left(\mathrm{NCCH}_{3}\right)\right]^{+}(\mathrm{m} / z=542.3)$.

The coordination of py onto $\mathrm{Ru}^{\mathrm{VI}} \equiv \mathrm{N}$ activates the complex toward $\mathrm{N} \cdots \mathrm{N}$ coupling or attack by nucleophiles. ${ }^{41,47} \mathrm{~A}$ similar activating effect by ligands has been observed in the epoxidation of alkenes by $\left[\mathrm{Cr}^{\mathrm{V}}(\text { salen })(\mathrm{O})\right]^{+}{ }^{6,49}$ In the presence of ligands such as pyridine $N$-oxide (pyO), the 6-coordinate species $\left[\mathrm{Cr}^{\mathrm{V}}(\text { salen })(\mathrm{O})(\mathrm{pyO})\right]^{+}$is formed, which reacts much more rapidly with alkenes to give epoxide and a (salen)Cr ${ }^{I I I}$ product. X-ray crystallographic studies indicate that in the 5 -coordinate $\left[\mathrm{Cr}^{\mathrm{V}}(\text { salen })(\mathrm{O})\right]^{+}$, the chromium atom is displaced $0.53 \AA$ above the mean salen plane. However, in the 6-coordinate $\left[\mathrm{Cr}^{\mathrm{V}}(\text { salen })(\mathrm{O})(\mathrm{pyO})\right]^{+}$, the chromium atom is pulled back to $0.26 \AA$. On the other hand, in 6-coordinate (salen) $\mathrm{Cr}{ }^{\mathrm{III}}$ complexes such as $\left[\mathrm{Cr}(\mathrm{salen})\left(\mathrm{OH}_{2}\right)_{2}\right]^{+}$, the chromium is only $0.077 \AA$ displaced from the salen plane. Although the $\mathrm{Cr}=\mathrm{O}$ bond length increases by only $0.01 \AA$, the $v(\mathrm{Cr}=\mathrm{O})$ stretch decreases from $1004 \mathrm{~cm}^{-1}$ to $943 \mathrm{~cm}^{-1}$ upon ligation, indicating a weakening of the $\mathrm{Cr}=\mathrm{O}$ bond. Hence, upon coordination by a good donor ligand, the $\mathrm{Cr}^{\mathrm{V}}=\mathrm{O}$ species becomes more product-like, and this should lower the reorganization energy prior to oxygen atom transfer. Similarly, in $\left[\mathrm{Ru}^{\mathrm{VI}}(\mathrm{N})\left(\mathrm{L}^{1}\right)(\mathrm{MeOH})\right]^{+}$the $\mathrm{Ru}$ atom is displaced $0.39 \AA$ from the mean $\mathrm{L}^{1}$ plane, ${ }^{41}$ whereas in a typical six-coordinate ruthenium(III) species such as $\left[\mathrm{Ru}^{\mathrm{III}}\left(\mathrm{L}^{1}\right)\left(\mathrm{NH}_{2} \mathrm{Ph}\right)_{2}\right]^{+}$, the ruthenium atom is only $0.075 \AA$ displaced from the $\mathrm{L}^{1}$ plane. Although we were unable to obtain the crystal structure or the IR spectrum of $\left[\mathrm{Ru}^{\mathrm{VI}}(\mathrm{N})\left(\mathrm{L}^{1}\right)(\mathrm{py})\right]^{+}$due to its high reactivity, it is reasonable to assume that upon pyridine coordination, the ruthenium atom is pulled back toward the $\mathrm{L}^{1}$ plane and the $\mathrm{Ru} \equiv \mathrm{N}$ bond is weakened.

This facile ligand-induced $\mathrm{N} \cdots \mathrm{N}$ coupling reaction is utilized in the preparation of a series of new ruthenium(III) salen complexes, as described in the next section.

Synthesis of Ruthenium(III) Salen Complexes. Treatment of $\left[\mathrm{Ru}^{\mathrm{VI}}(\mathrm{N})\left(\mathrm{L}^{1}\right)(\mathrm{MeOH})\right]^{+}$(1) with various ligands affords a series of $\mathrm{Ru}^{\mathrm{III}} \mathrm{L}^{1}$ complexes, as illustrated in Scheme 2.

The diaquo species $\left[\mathrm{Ru}^{\mathrm{III}}\left(\mathrm{L}^{1}\right)\left(\mathrm{OH}_{2}\right)_{2}\right]\left(\mathrm{PF}_{6}\right)(\mathbf{2})$, which is readily prepared from $\mathbf{1}$ by refluxing in water/acetone, ${ }^{43}$ is also a useful starting material for preparing various $\mathrm{Ru}^{\mathrm{III}} \mathrm{L}$ complexes. 2-9 have room temperature magnetic moments (solid sample, Gouy method) of $\mu_{\text {eff }}=1.86-2.07 \mu_{\mathrm{B}}$, consistent with their formulation as low-spin $\mathrm{d}^{5}$ ruthenium(III) complexes. Their $\mathrm{UV}-$ vis spectra in $\mathrm{CH}_{3} \mathrm{CN}$ (Table 2) display a broad band at $645-734 \mathrm{~nm}$, which is assigned to salen to a $\mathrm{Ru}^{\mathrm{III}}$ LMCT transition. ${ }^{15}$ There are also intense peaks at $332-415 \mathrm{~nm}$, which can be attributed to intraligand transitions of the coordinated salen ligand. The IR spectra of 2-9 display sharp $v(C=\mathrm{N})$ stretches at around $1600 \mathrm{~cm}^{-1}$. The IR spectrum of 5 shows a peak at $1017 \mathrm{~cm}^{-1}$, which is absent in other $\mathrm{Ru}^{\mathrm{III}} \mathrm{L}^{1}$ complexes, and this is assigned to $v(\mathrm{~S}=\mathrm{O})$ stretch of $\mathrm{S}$-bonded $\mathrm{Me}_{2} \mathrm{SO}$. $v(\mathrm{~S}=\mathrm{O})$ stretch of $\mathrm{S}$-bonded $\mathrm{Ru}-\mathrm{Me}_{2} \mathrm{SO}$ complexes typically occur

(49) Siddall, T. L.; Miyaura, N.; Huffman, J. C.; Kochi, J. K. J. Chem. Soc., Chem. Commun. 1983, 1185-1186. 
Scheme 2. Synthesis of $\mathrm{Ru}^{\mathrm{III}} \mathrm{L}^{1}$ Complexes via Ligand-Induced $\mathrm{N} \cdots \mathrm{N}$ Coupling of the Corresponding Ruthenium(VI) Nitride

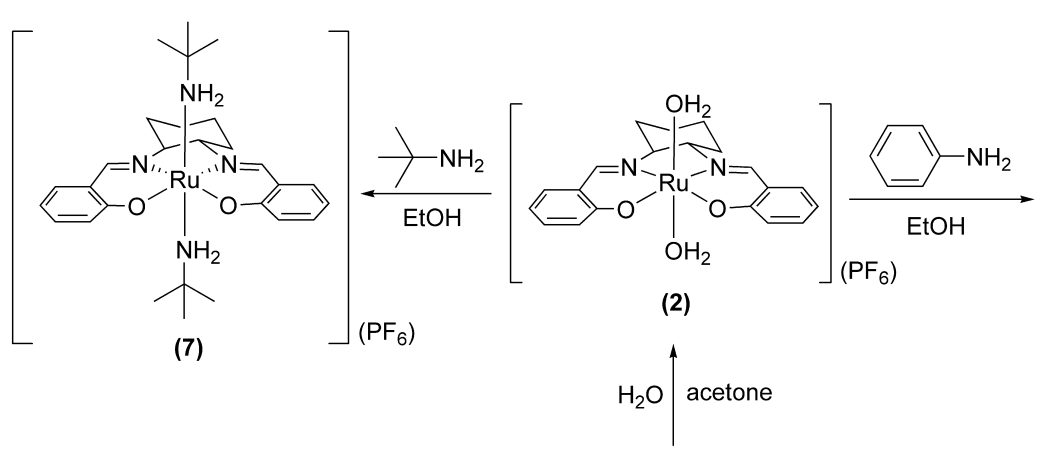

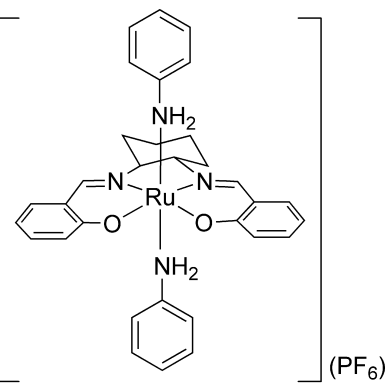

(6)
(K)

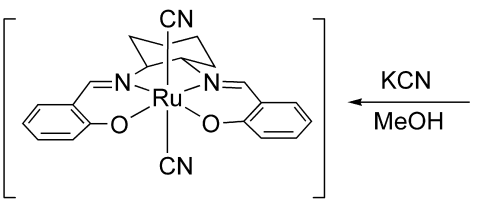

(9)

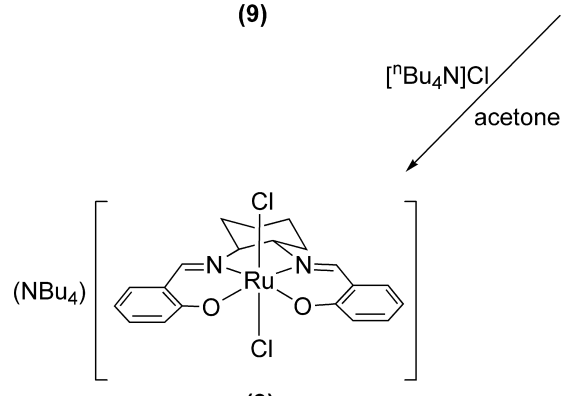

(8)

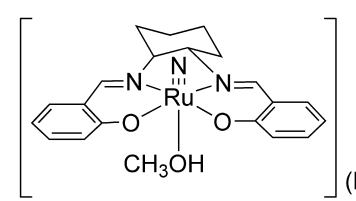

(1)<smiles>CS(C)=O</smiles>

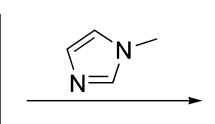
$\left(\mathrm{PF}_{6}\right)$<smiles>Cc1ccccn1</smiles>

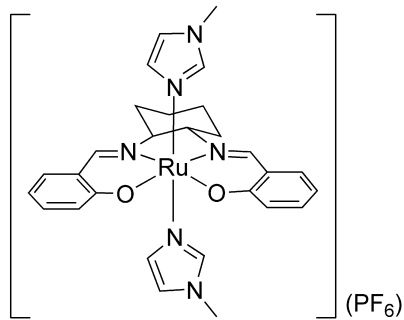

(3)

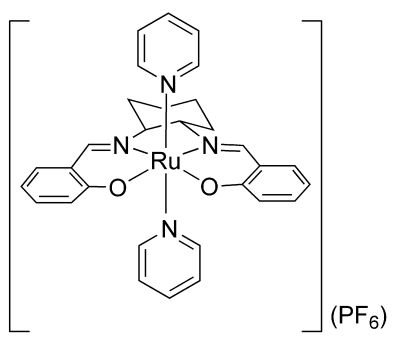

(4)
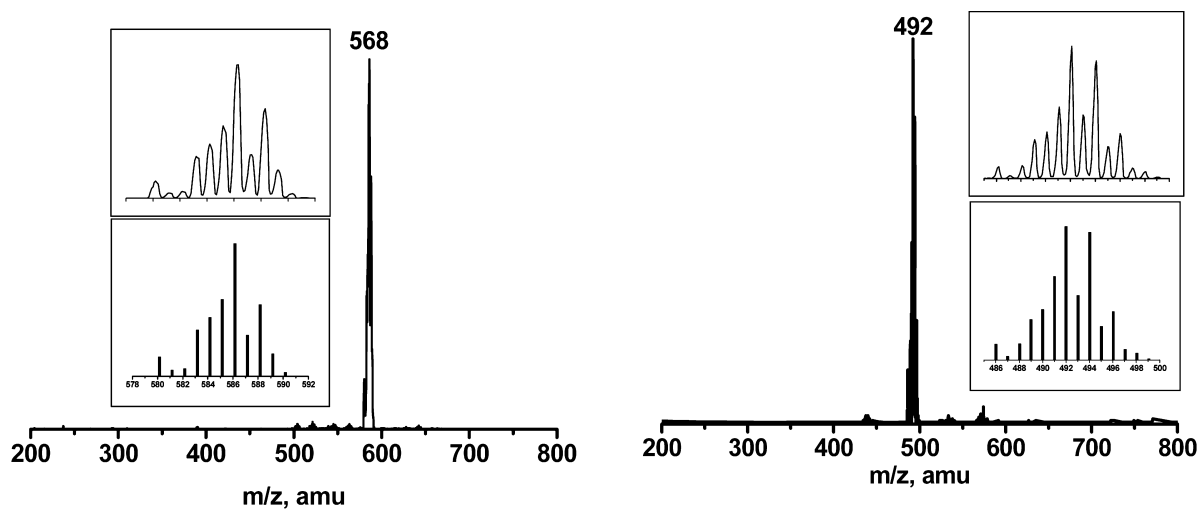

Figure 3. ESI mass spectra of $\mathbf{3}$ in the positive mode (left) and $\mathbf{8}$ in the negative mode (right) in acetone. Insets show the experimental (top) and calculated (bottom) isotopic patterns.

at $1020-1134 \mathrm{~cm}^{-1}$, whereas that of O-bonded $\mathrm{Ru}-\mathrm{Me}_{2} \mathrm{SO}$ complexes occur at lower wave numbers (885-954 $\left.\mathrm{cm}^{-1}\right) .{ }^{50-53}$ This assignment is also supported by electrochemical studies (below). The $v(\mathrm{~N}-\mathrm{H})$ stretches for the

(50) Reisner, E.; Arion, V. B.; Guedes da Silva, M. F. C.; Lichtenecker, R.; Eichinger, A.; Keppler, B. K.; Kukushkin, V. Y.; Pombeiro, A. J. L. Inorg. Chem. 2004, 43, 7083-7093.

(51) Alessio, E.; Mestroni, G.; Nardin, G.; Attia, W. M.; Calligaris, M.; Sava, G.; Zorzet, S. Inorg. Chem. 1988, 27, 4099-4106.

(52) Alessio, E.; Bolle, M.; Milani, B.; Mestroni, G.; Faleschini, P.; Geremia, S.; Calligaris, M. Inorg. Chem. 1995, 34, 4716-4721.

(53) Alessio, E.; Milani, B.; Bolle, M.; Mestroni, G.; Faleschini, P.; Todone, F.; Geremia, S.; Calligaris, M. Inorg. Chem. 1995, 34, 4722-4734.

(54) Yeung, W. F.; Man, W. L.; Wong, W. T.; Lau, T. C.; Gao, S. Angew. Chem., Int. Ed. 2001, 40, 3031-3033. amines 6 and 7 occur at 3311, 3278 and 3300, $3244 \mathrm{~cm}^{-1}$ respectively. The $v(\mathrm{C} \equiv \mathrm{N})$ stretch at $2090 \mathrm{~cm}^{-1}$ for 9 is similar to that of trans-[Ru$\left.{ }^{\mathrm{III}}(\mathrm{acac})_{2}(\mathrm{CN})_{2}\right]^{-}\left(2099 \mathrm{~cm}^{-1}\right)^{54}$ and trans-[Ru $\mathrm{Ru}^{\mathrm{III}}($ salen $\left.)(\mathrm{CN})_{2}\right]^{-}\left(2080 \mathrm{~cm}^{-1}\right) .^{55}$

The ESI-MS of 2-9 in acetone exhibit a peak due to the parent ion (Experimental Section). For example, the ESI mass spectra of $\mathbf{3}$ (positive mode) and $\mathbf{8}$ (negative mode) (Figure 3) show one single peak that arises from the parent ion $\left[\mathrm{Ru}\left(\mathrm{L}^{1}\right)(1-\mathrm{MeIm})_{2}\right]^{+}(\mathrm{m} / \mathrm{z}=568)$ and $\left[\mathrm{Ru}\left(\mathrm{L}^{1}\right) \mathrm{Cl}_{2}\right]^{-}(\mathrm{m} / z=$ 492), respectively.

(55) Yeung, W. F.; Lau, P. H.; Lau, T. C.; Wei, H. Y.; Sun, H. L.; Gao, S.; Chen, Z. D.; Wong, W. T. Inorg. Chem. 2005, 44, 6579-6590. 


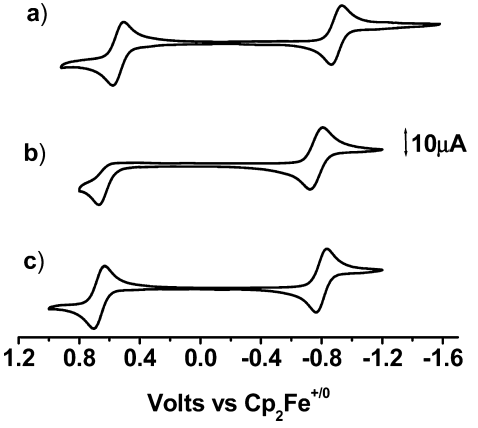

Figure 4. Cyclic voltammograms of $\mathbf{3}$ (a), 6 (b), and 7 (c) in $\mathrm{CH}_{3} \mathrm{CN}$.

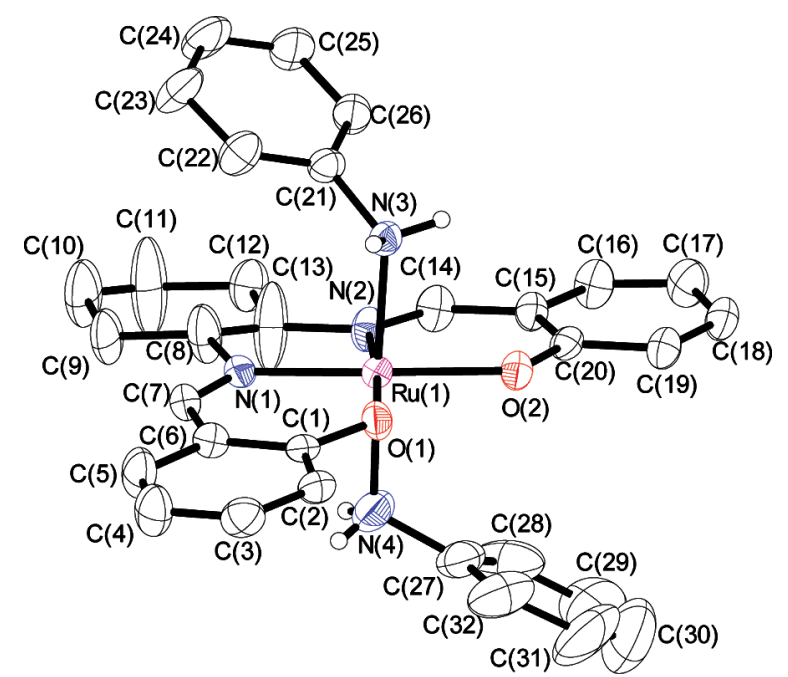

Figure 5. ORTEP diagram of $\left[\mathrm{Ru}^{\mathrm{III}}\left(\mathrm{L}^{1}\right)\left(\mathrm{NH}_{2} \mathrm{Ph}\right)_{2}\right]^{+}$cation (6); thermal ellipsoids are drawn at $30 \%$ probability (hydrogen atoms are omitted except $\mathrm{N}(3)-\mathrm{H}$ and $\mathrm{N}(4)-\mathrm{H}$ for clarity).

The cyclic voltammograms of $\mathbf{3}-\mathbf{8}$ in $\mathrm{CH}_{3} \mathrm{CN}$ (Table 2) exhibit two reversible couples, which are assigned to $\mathrm{Ru}^{\mathrm{IV} / I I I}$ and $\mathrm{Ru}^{\mathrm{IIIIII}}$ couples, except for $\mathbf{5}$ and $\mathbf{6}$ where the oxidation waves are irreversible (Figure 4). As expected, compounds with anionic axial ligands $(\mathbf{8}, \mathbf{1 2})$ have lower $\mathrm{Ru}^{\mathrm{IV} / I I I}$ and $\mathrm{Ru}^{\mathrm{III} / \mathrm{II}}$ potentials. For a compound with neutral axial ligands, the $\mathrm{Ru}^{\mathrm{III} / \mathrm{II}}$ potential increases with the $\pi$-accepting ability of the ligand. 5 has the highest $\mathrm{Ru}^{\mathrm{III} / \mathrm{II}}$ potential, consistent with evidence from IR that the $\mathrm{Me}_{2} \mathrm{SO}$ ligands are S-bonded; $\mathrm{S}$-bonded $\mathrm{Me}_{2} \mathrm{SO}$ is a strong $\pi$-acceptor ligand that would stabilize $\mathrm{Ru}^{\mathrm{II}}$, whereas the O-bonded ligand would have little $\pi$-accepting ability. ${ }^{56}$

The molecular structures of $\mathbf{6}$ (Figure 5) and 9 (Figure 6) have been determined by single-crystal X-ray diffraction method. The crystal data and structural refinement details are given in Table 1. Selected bond distances and angles are listed in Table 3 and 4. 6 and $\mathbf{9}$ adopt a distorted octahedral geometry; the ruthenium center is bonded to the two oxygen atoms and the two nitrogen atoms of the $\mathrm{L}^{1}$ ligand in the equatorial plane. They have similar $\mathrm{Ru}-\mathrm{O}$ bond distances (2.027(4) and 2.029(4) $\AA$ for 6, 2.018(2) and 2.022(2) $\AA$ for 9) and $\mathrm{Ru}-\mathrm{N}$ bond distances (1.986(5) and 1.982(6) $\AA$ for $\mathbf{6},(1.996(2)$ and 1.993(2) $\AA$ for 9). 6 is the first crystal structure of a ruthenium(III) complex of $\mathrm{PhNH}_{2}$. Its $\mathrm{Ru}-\mathrm{N}_{\text {aniline }}$ bond distances of 2.126(5) and 2.141(12) $\AA$ are slightly shorter than that of the ruthenium(II) species $\left[\mathrm{Ru}^{\mathrm{II}}\left(\mathrm{NH}_{2} \mathrm{Ph}\right)\{\mathrm{PhNC}(\mathrm{H}) \mathrm{NPh}\}\left(\mathrm{Me}_{2} \mathrm{SO}\right)_{2} \mathrm{Cl}\right](2.1907(19) \AA) .{ }^{57}$

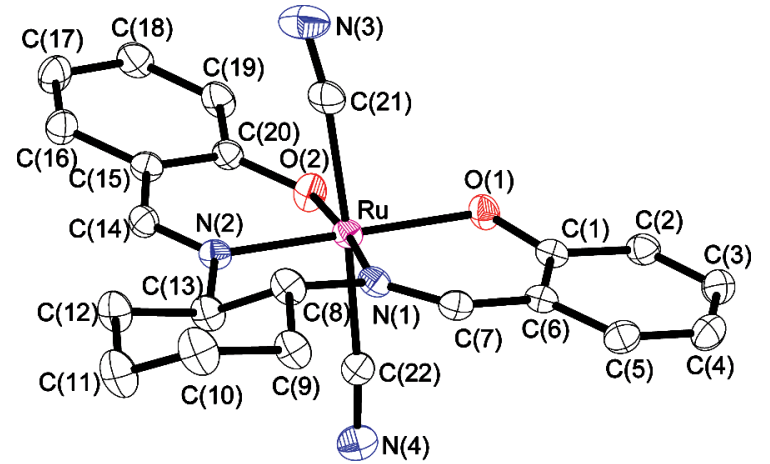

Figure 6. ORTEP diagram of $\left[\mathrm{Ru}^{\mathrm{III}}\left(\mathrm{L}^{1}\right)(\mathrm{CN})_{2}\right]^{-}$anion (9), thermal ellipsoids are drawn at $30 \%$ probability (hydrogen atoms are omitted for clarity).

Table 3. Selected Bond Distances (Angstroms) and Angles (Degrees) of 6

\begin{tabular}{lrlr}
\hline $\mathrm{Ru}(1)-\mathrm{O}(1)$ & $2.029(5)$ & $\mathrm{Ru}(1)-\mathrm{N}(3)$ & $2.126(5)$ \\
$\mathrm{Ru}(1)-\mathrm{O}(2)$ & $2.027(4)$ & $\mathrm{Ru}(1)-\mathrm{N}(4)$ & $2.141(12)$ \\
$\mathrm{Ru}(1)-\mathrm{N}(1)$ & $1.986(5)$ & $\mathrm{N}(1)-\mathrm{C}(7)$ & $1.291(9)$ \\
$\mathrm{Ru}(1)-\mathrm{N}(2)$ & $1.982(6)$ & $\mathrm{N}(2)-\mathrm{C}(14)$ & $1.287(9)$ \\
$\mathrm{O}(1)-\mathrm{Ru}(1)-\mathrm{O}(2)$ & $91.25(17)$ & $\mathrm{N}(1)-\mathrm{Ru}(1)-\mathrm{N}(2)$ & $83.3(2)$ \\
$\mathrm{O}(1)-\mathrm{Ru}(1)-\mathrm{N}(1)$ & $92.8(2)$ & $\mathrm{N}(1)-\mathrm{Ru}(1)-\mathrm{N}(3)$ & $94.3(2)$ \\
$\mathrm{O}(1)-\mathrm{Ru}(1)-\mathrm{N}(2)$ & $176.0(2)$ & $\mathrm{N}(1)-\mathrm{Ru}(1)-\mathrm{N}(4)$ & $89.4(6)$ \\
$\mathrm{O}(1)-\mathrm{Ru}(1)-\mathrm{N}(3)$ & $86.6(2)$ & $\mathrm{N}(2)-\mathrm{Ru}(1)-\mathrm{N}(3)$ & $94.8(2)$ \\
$\mathrm{O}(1)-\mathrm{Ru}(1)-\mathrm{N}(4)$ & $92.7(7)$ & $\mathrm{N}(2)-\mathrm{Ru}(1)-\mathrm{N}(4)$ & $86.2(7)$ \\
$\mathrm{O}(2)-\mathrm{Ru}(1)-\mathrm{N}(1)$ & $175.9(2)$ & $\mathrm{N}(3)-\mathrm{Ru}(1)-\mathrm{N}(4)$ & $176.3(5)$ \\
$\mathrm{O}(2)-\mathrm{Ru}(1)-\mathrm{N}(2)$ & $92.6(2)$ & $\mathrm{Ru}(1)-\mathrm{N}(1)-\mathrm{C}(7)$ & $125.9(5)$ \\
$\mathrm{O}(2)-\mathrm{Ru}(1)-\mathrm{N}(3)$ & $86.7(2)$ & $\mathrm{Ru}(1)-\mathrm{N}(2)-\mathrm{C}(14)$ & $126.1(5)$ \\
$\mathrm{O}(2)-\mathrm{Ru}(1)-\mathrm{N}(4)$ & $89.7(6)$ & &
\end{tabular}

Table 4. Selected Bond Distances (Angstroms) and Angles (Degrees) of 9

\begin{tabular}{lclr}
\hline $\mathrm{Ru}(1)-\mathrm{O}(1)$ & $2.018(2)$ & $\mathrm{Ru}(1)-\mathrm{C}(22)$ & $2.067(4)$ \\
$\mathrm{Ru}(1)-\mathrm{O}(2)$ & $2.022(2)$ & $\mathrm{N}(1)-\mathrm{C}(7)$ & $1.286(4)$ \\
$\mathrm{Ru}(1)-\mathrm{N}(1)$ & $1.996(2)$ & $\mathrm{N}(2)-\mathrm{C}(14)$ & $1.290(4)$ \\
$\mathrm{Ru}(1)-\mathrm{N}(2)$ & $1.993(2)$ & $\mathrm{C}(21)-\mathrm{N}(3)$ & $1.157(4)$ \\
$\mathrm{Ru}(1)-\mathrm{C}(21)$ & $2.076(4)$ & $\mathrm{C}(22)-\mathrm{N}(4)$ & $1.147(4)$ \\
$\mathrm{O}(1)-\mathrm{Ru}(1)-\mathrm{O}(2)$ & $91.82(8)$ & $\mathrm{N}(1)-\mathrm{Ru}(1)-\mathrm{N}(2)$ & $83.28(10)$ \\
$\mathrm{O}(1)-\mathrm{Ru}(1)-\mathrm{N}(1)$ & $92.67(9)$ & $\mathrm{N}(1)-\mathrm{Ru}(1)-\mathrm{C}(21)$ & $94.77(4)$ \\
$\mathrm{O}(1)-\mathrm{Ru}(1)-\mathrm{N}(2)$ & $175.56(9)$ & $\mathrm{N}(1)-\mathrm{Ru}(1)-\mathrm{C}(22)$ & $88.55(11)$ \\
$\mathrm{O}(1)-\mathrm{Ru}(1)-\mathrm{C}(21)$ & $92.93(11)$ & $\mathrm{N}(2)-\mathrm{Ru}(1)-\mathrm{C}(21)$ & $85.63(12)$ \\
$\mathrm{O}(1)-\mathrm{Ru}(1)-\mathrm{C}(22)$ & $90.56(11)$ & $\mathrm{N}(2)-\mathrm{Ru}(1)-\mathrm{C}(22)$ & $91.15(12)$ \\
$\mathrm{O}(2)-\mathrm{Ru}(1)-\mathrm{N}(1)$ & $174.90(9)$ & $\mathrm{C}(21)-\mathrm{Ru}(1)-\mathrm{C}(22)$ & $175.07(13)$ \\
$\mathrm{O}(2)-\mathrm{Ru}(1)-\mathrm{N}(2)$ & $92.31(9)$ & $\mathrm{Ru}(1)-\mathrm{N}(1)-\mathrm{C}(7)$ & $124.9(2)$ \\
$\mathrm{O}(2)-\mathrm{Ru}(1)-\mathrm{C}(21)$ & $87.40(11)$ & $\mathrm{Ru}(1)-\mathrm{N}(2)-\mathrm{C}(14)$ & $124.8(2)$ \\
$\mathrm{O}(2)-\mathrm{Ru}(1)-\mathrm{C}(22)$ & $89.00(11)$ & &
\end{tabular}

In 9, the $\mathrm{Ru}-\mathrm{C}_{\text {cyanide }}$ bond distances (2.067(4) and 2.075(4) $\AA)$ are similar to that in $\left(\mathrm{N}^{n} \mathrm{Bu}_{4}\right)\left[\mathrm{Ru}^{\mathrm{III}}(\right.$ salen $\left.)(\mathrm{CN})_{2}\right](2.04(2)$ and 2.09(2) $\AA$ )..$^{55}$

We have previously used $\left[\mathrm{Au}(\mathrm{CN})_{2}\right]^{-}$as a bridging ligand for the construction of coordination polymers. ${ }^{58,59}$ Our initial goal in this study was to use $\left[\mathrm{Au}(\mathrm{CN})_{2}\right]^{-}$as a ligand to induce $\mathrm{N} \cdots \mathrm{N}$ coupling, which may result in the formation of a polynuclear species. However, treatment of $\left[\mathrm{Ru}^{\mathrm{VI}}(\mathrm{N})\left(\mathrm{L}^{2}\right) \mathrm{Cl}\right]$ with $\mathrm{K}\left[\mathrm{Au}(\mathrm{CN})_{2}\right]$ in $\mathrm{CH}_{3} \mathrm{OH} / \mathrm{H}_{2} \mathrm{O}$ did not result in the coordination of $\left[\mathrm{Au}(\mathrm{CN})_{2}\right]^{-}$onto ruthenium, instead $\left[\mathrm{Ru}^{\mathrm{III}}\left(\mathrm{L}^{2}\right)\left(\mathrm{NCCH}_{3}\right)_{2}\right]\left[\mathrm{Au}(\mathrm{CN})_{2}\right](\mathbf{1 1})$ was isolated after re-

(56) Wishart, J. F.; Taube, H.; Breslauer, K. J.; Isied, S. S. Inorg. Chem. 1984, 23, 2997-3001.

(57) Clark, T.; Cochrane, J.; Colson, S. F.; Malik, K. Z.; Robinson, S. D.; Steed, J. W. Polyhedron 2001, 20, 1875-1880.

(58) Yeung, W. F.; Wong, W. T.; Zuo, J. L.; Lau, T. C. J. Chem. Soc., Dalton Trans. 2000, 629-631.

(59) Shek, I. P. Y.; Wong, W. Y.; Lau, T. C. New J. Chem. 2000, 24, 733-734.

(60) Demerseman, B.; Renaud, J. L.; Toupet, L.; Hubert, C.; Bruneau, C. Eur. J. Inorg. Chem. 2006, 7, 1371-1380. 


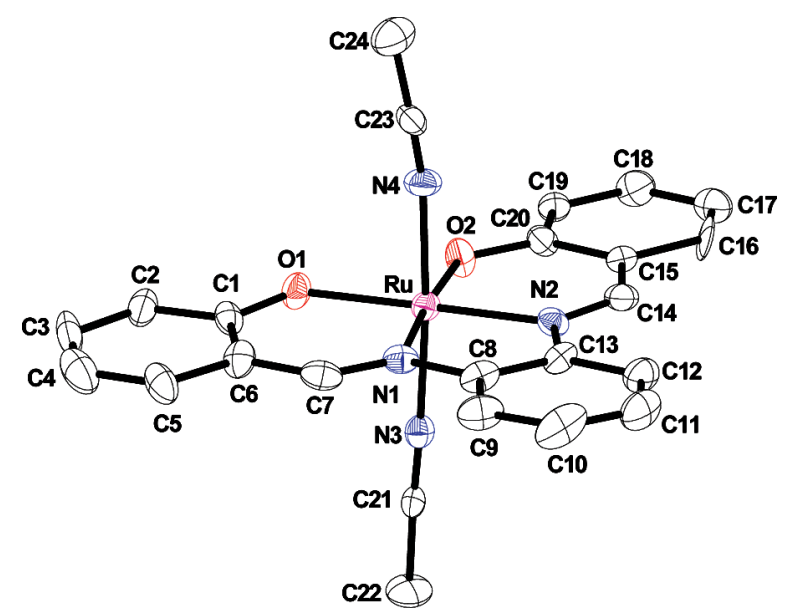

Figure 7. ORTEP diagram of $\left[\mathrm{Ru}^{\mathrm{III}}\left(\mathrm{L}^{2}\right)\left(\mathrm{NCCH}_{3}\right)_{2}\right]^{+}$cation (11), thermal ellipsoids are drawn at $30 \%$ probability (hydrogen atoms are omitted for clarity).

Table 5. Selected Bond Distances (Angstroms) and Angles (Degrees) of 11

\begin{tabular}{lrlc}
\hline $\mathrm{Ru}(1)-\mathrm{O}(1)$ & $2.011(6)$ & $\mathrm{Ru}(1)-\mathrm{N}(4)$ & $2.040(9)$ \\
$\mathrm{Ru}(1)-\mathrm{O}(2)$ & $2.002(6)$ & $\mathrm{N}(1)-\mathrm{C}(7)$ & $1.305(13)$ \\
$\mathrm{Ru}(1)-\mathrm{N}(1)$ & $1.992(8)$ & $\mathrm{N}(2)-\mathrm{C}(14)$ & $1.297(12)$ \\
$\mathrm{Ru}(1)-\mathrm{N}(2)$ & $2.016(8)$ & $\mathrm{N}(3)-\mathrm{C}(21)$ & $1.145(13)$ \\
$\mathrm{Ru}(1)-\mathrm{N}(3)$ & $2.041(9)$ & $\mathrm{N}(4)-\mathrm{C}(22)$ & $1.111(15)$ \\
$\mathrm{O}(1)-\mathrm{Ru}(1)-\mathrm{O}(2)$ & $90.4(3)$ & $\mathrm{N}(1)-\mathrm{Ru}(1)-\mathrm{N}(2)$ & $82.4(3)$ \\
$\mathrm{O}(1)-\mathrm{Ru}(1)-\mathrm{N}(1)$ & $93.8(3)$ & $\mathrm{N}(1)-\mathrm{Ru}(1)-\mathrm{N}(3)$ & $88.0(4)$ \\
$\mathrm{O}(1)-\mathrm{Ru}(1)-\mathrm{N}(2)$ & $175.9(3)$ & $\mathrm{N}(1)-\mathrm{Ru}(1)-\mathrm{N}(4)$ & $89.8(4)$ \\
$\mathrm{O}(1)-\mathrm{Ru}(1)-\mathrm{N}(3)$ & $88.6(3)$ & $\mathrm{N}(2)-\mathrm{Ru}(1)-\mathrm{N}(3)$ & $92.8(4)$ \\
$\mathrm{O}(1)-\mathrm{Ru}(1)-\mathrm{N}(4)$ & $88.1(4)$ & $\mathrm{N}(2)-\mathrm{Ru}(1)-\mathrm{N}(4)$ & $90.4(4)$ \\
$\mathrm{O}(2)-\mathrm{Ru}(1)-\mathrm{N}(1)$ & $175.4(3)$ & $\mathrm{N}(3)-\mathrm{Ru}(1)-\mathrm{N}(4)$ & $175.9(4)$ \\
$\mathrm{O}(2)-\mathrm{Ru}(1)-\mathrm{N}(2)$ & $93.5(3)$ & $\mathrm{Ru}(1)-\mathrm{N}(3)-\mathrm{C}(21)$ & $177.1(9)$ \\
$\mathrm{O}(2)-\mathrm{Ru}(1)-\mathrm{N}(3)$ & $90.2(4)$ & $\mathrm{Ru}(1)-\mathrm{N}(4)-\mathrm{C}(23)$ & $169.6(11)$ \\
$\mathrm{O}(2)-\mathrm{Ru}(1)-\mathrm{N}(4)$ & $92.2(4)$ & &
\end{tabular}

crystallization from $\mathrm{CH}_{3} \mathrm{CN} / \mathrm{H}_{2} \mathrm{O}$. It shows a peak at 2140 $\mathrm{cm}^{-1}$ in the IR, which is assigned to the $v(\mathrm{C} \equiv \mathrm{N})$ stretch of $\left[\mathrm{Au}(\mathrm{CN})_{2}\right]^{-}$. The $\left[\mathrm{Au}(\mathrm{CN})_{2}\right]^{-}$functions only as a counterion, in fact $\left[\mathrm{Ru}^{\mathrm{III}}\left(\mathrm{L}^{2}\right)\left(\mathrm{NCCH}_{3}\right)_{2}\right] \mathrm{PF}_{6}$ can be obtained by refluxing $\left[\mathrm{Ru}^{\mathrm{VI}}(\mathrm{N})\left(\mathrm{L}^{2}\right)\left(\mathrm{CH}_{3} \mathrm{OH}\right)\right]\left(\mathrm{ClO}_{4}\right)$ in $\mathrm{CH}_{3} \mathrm{CN}$. In both compounds the $v(\mathrm{C} \equiv \mathrm{N})$ stretch of the coordinated $\mathrm{CH}_{3} \mathrm{CN}$ is too weak to be observed. The X-ray crystal structure of $\mathbf{1 1}$ is shown in Figure 7, and the details of the crystal data and selected bond data are listed in Tables 1 and 5, respectively. The two $\mathrm{CH}_{3} \mathrm{CN}$ ligands are trans with a $\mathrm{N}(3)-\mathrm{Ru}-\mathrm{N}(4)$ angle of $175.9(4)^{\circ}$. The two $\mathrm{Ru}-\mathrm{N}_{\text {acetonitrile }}$ bond distances are identical (2.040(9) and 2.041 (9) $\AA$ ), and are comparable to that of $\left[\mathrm{Ru}^{\mathrm{II}}(\mathrm{Cp} *)\left(\mathrm{Ph}_{2} \mathrm{POMe}\right)\left(\mathrm{NCCH}_{3}\right)_{2}\right](2.067(2) \text { and } 2.076(2) \AA)^{60}$ and $m e r-\left[\mathrm{Ru}^{\mathrm{III}}\left(\mathrm{NCCH}_{3}\right)_{3} \mathrm{Cl}_{3}\right](2.014(7)-2.065(7) \AA) .{ }^{61}$

Attempts to prepare a ruthenium(VI) nitrido complex of the parent salen ligand by a reaction of $\left[\mathrm{N}^{n} \mathrm{Bu}_{4}\right]\left[\mathrm{Ru}^{\mathrm{VI}}(\mathrm{N}) \mathrm{Cl}_{4}\right]$ with $\mathrm{H}_{2} \mathrm{~L}^{3}$ was unsuccessful; instead the dark-green complex $\left[\mathrm{N}^{n} \mathrm{Bu}_{4}\right]\left[\mathrm{Ru}^{\mathrm{III}}\left(\mathrm{L}^{3}\right) \mathrm{Cl}_{2}\right]$ (12) was obtained. 12 has a roomtemperature magnetic moment of $\mu_{\mathrm{eff}}=1.79 \mu_{\mathrm{B}}$ (solid sample, Gouy method) consistent with its formulation as a low-spin $\mathrm{d}^{5} \mathrm{Ru}^{\mathrm{III}}$ complex. The ESI mass spectrum (-ve mode) of $\mathbf{1 2}$ in acetone shows a single peak at $\mathrm{m} / \mathrm{z}=438$, which is assigned to the parent ion $\left[\mathrm{Ru}^{\mathrm{III}}\left(\mathrm{L}^{3}\right) \mathrm{Cl}_{2}\right]^{-}$. The cyclic voltammogram of $\mathbf{1 2}$ (Figure 8) in $\mathrm{CH}_{3} \mathrm{CN}$ shows a reversible oxidation wave at $E_{1 / 2}=+0.16 \mathrm{~V}\left(\mathrm{vs}_{\mathrm{Cp}_{2}} \mathrm{Fe}^{+/ 0}\right)$ and an irreversible reduction wave at $E_{\mathrm{pa}}=-1.39 \mathrm{~V}\left(\mathrm{vs} \mathrm{Cp}_{2} \mathrm{Fe}^{+/ 0}\right)$, which are assigned to $\mathrm{Ru}^{\mathrm{IV} / I I I}$ and $\mathrm{Ru}^{\mathrm{III} / \mathrm{II}}$ couples, respectively.

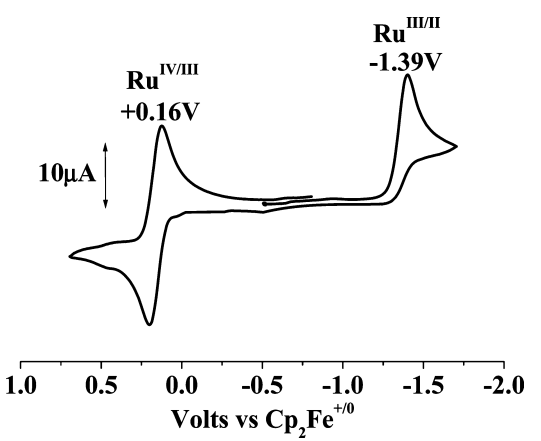

Figure 8. Cyclic voltammogram of $\mathbf{1 2}$ in $\mathrm{CH}_{3} \mathrm{CN}$.

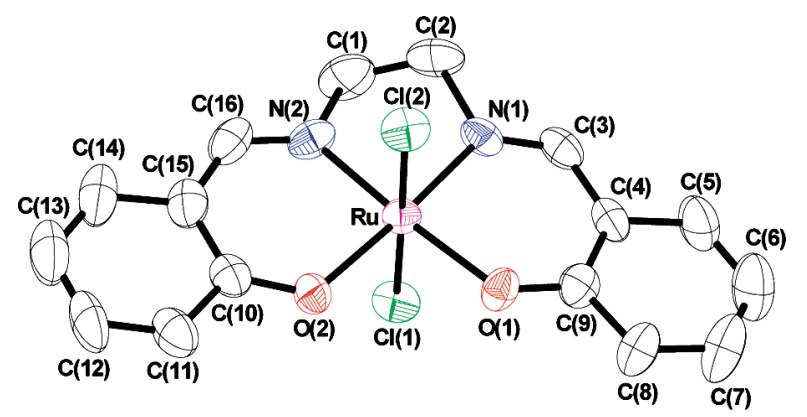

Figure 9. ORTEP diagram of $\left[\mathrm{Ru}^{\mathrm{III}}\left(\mathrm{L}^{3}\right) \mathrm{Cl}_{2}\right]^{-}$anion (12), thermal ellipsoids are drawn at $30 \%$ probability (hydrogen atoms are omitted for clarity).

Table 6. Selected Bond Distances (Angstroms) and Angles (Degrees) of 12

\begin{tabular}{lclc}
\hline $\mathrm{Ru}(1)-\mathrm{O}(1)$ & $2.029(6)$ & $\mathrm{Ru}(1)-\mathrm{Cl}(1)$ & $2.386(2)$ \\
$\mathrm{Ru}(1)-\mathrm{O}(2)$ & $2.040(6)$ & $\mathrm{Ru}(1)-\mathrm{Cl}(2)$ & $2.378(2)$ \\
$\mathrm{Ru}(1)-\mathrm{N}(1)$ & $1.984(7)$ & $\mathrm{N}(1)-\mathrm{C}(3)$ & $1.283(11)$ \\
$\mathrm{Ru}(1)-\mathrm{N}(2)$ & $1.992(8)$ & $\mathrm{N}(2)-\mathrm{C}(16)$ & $1.270(12)$ \\
$\mathrm{O}(1)-\mathrm{Ru}(1)-\mathrm{O}(2)$ & $91.9(2)$ & $\mathrm{O}(2)-\mathrm{Ru}(1)-\mathrm{Cl}(2)$ & $89.18(18)$ \\
$\mathrm{O}(1)-\mathrm{Ru}(1)-\mathrm{N}(1)$ & $92.2(3)$ & $\mathrm{N}(1)-\mathrm{Ru}(1)-\mathrm{N}(2)$ & $83.8(4)$ \\
$\mathrm{O}(1)-\mathrm{Ru}(1)-\mathrm{N}(2)$ & $175.9(3)$ & $\mathrm{N}(1)-\mathrm{Ru}(1)-\mathrm{Cl}(1)$ & $88.2(2)$ \\
$\mathrm{O}(1)-\mathrm{Ru}(1)-\mathrm{Cl}(1)$ & $89.66(19)$ & $\mathrm{N}(1)-\mathrm{Ru}(1)-\mathrm{Cl}(2)$ & $91.7(2)$ \\
$\mathrm{O}(1)-\mathrm{Ru}(1)-\mathrm{Cl}(2)$ & $91.12(19)$ & $\mathrm{N}(2)-\mathrm{Ru}(1)-\mathrm{Cl}(1)$ & $90.4(2)$ \\
$\mathrm{O}(2)-\mathrm{Ru}(1)-\mathrm{N}(1)$ & $175.8(3)$ & $\mathrm{N}(2)-\mathrm{Ru}(1)-\mathrm{Cl}(2)$ & $88.8(2)$ \\
$\mathrm{O}(2)-\mathrm{Ru}(1)-\mathrm{N}(2)$ & $92.2(3)$ & $\mathrm{Cl}(1)-\mathrm{Ru}(1)-\mathrm{Cl}(2)$ & $179.22(10)$ \\
$\mathrm{O}(2)-\mathrm{Ru}(1)-\mathrm{Cl}(1)$ & $90.83(18)$ & &
\end{tabular}

The potentials are comparable to that of $\mathbf{8}$ (Table 2). The irreversibility of the $\mathrm{Ru}^{\mathrm{III} / \mathrm{II}}$ couple may be due to chloride dissociation in the $\mathrm{Ru}^{\mathrm{II}}$ state.

Single crystals suitable for X-ray crystallography were obtained by slow diffusion of diethyl ether into a $\mathrm{CH}_{2} \mathrm{Cl}_{2}$ solution of 12. The molecular structure of the anion is shown in Figure 9. Crystal data, structural refinement details, and selected bond distances and angles are given in Tables 1 and 6. The two chlorine atoms are trans with $\mathrm{Cl}(1)-\mathrm{Ru}-\mathrm{Cl}(2)$ angle of $179.22(10)^{\circ}$. The two $\mathrm{Ru}-\mathrm{Cl}$ bond distances are identical (2.386(2) and 2.378(2) $\AA$ ) and are comparable to those in trans $-\mathrm{PPh}_{4}\left[\mathrm{Ru}(\mathrm{acac})_{2} \mathrm{Cl}_{2}\right]$ (2.355(2) and 2.362(1) A). ${ }^{62}$

\section{Conclusions}

Ruthenium(VI) nitrido complexes bearing salen-type ligands readily undergo facile ligand-induced $\mathrm{N} \cdots \mathrm{N}$ coupling reac-

(61) Appelbaum, L.; Heinrichs, C.; Demtschuk, J.; Michman, M.; Oron, M.; Schafer, H. J.; Schumann, H. J. Organomet. Chem. 1999, 592, 240-250.

(62) Hasegawa, T.; Lau, T. C.; Taube, H.; Schaefer, W. P. Inorg. Chem. 1991, 30, 2921-2928. 
tions, which provide a general synthetic pathway for a variety of (salen)ruthenium(III) complexes. Our studies should greatly increase the scope for the application of ruthenium salen complexes as catalysts for various organic transformations. The catalytic properties of some of these complexes will be investigated.

Acknowledgment. The work described in this article was supported by the Research Grants Council of Hong Kong
(CityU 2/06C, CityU 101404) and the City University of Hong Kong (7001799).

Supporting Information Available: UV-vis spectra of 3-9 and the ESI-MS of reaction of (1) and py in $\mathrm{CH}_{3} \mathrm{CN}$ taken in 5 $\mathrm{min}$. This material is available free of charge via the Internet at http://pubs.acs.org.

IC800263N 